\title{
A Numerical Investigation of the Eyewall Evolution in a Landfalling Typhoon
}

\author{
Chun-Chieh Wu and Hsiu-Ju Cheng \\ Department of Atmospheric Sciences, National Taiwan University, Taipei, Taiwan \\ YUQING WANG \\ International Pacific Research Center and Department of Meteorology, School of Ocean and Earth Science and Technology, \\ University of Hawaii at Manoa, Honolulu, Hawaii \\ Kun-Hsuan ChOU \\ Department of Atmospheric Sciences, Chinese Culture University, Taipei, Taiwan
}

(Manuscript received 9 January 2008, in final form 27 June 2008)

\begin{abstract}
An interesting eyewall evolution occurred in Typhoon Zeb (1998) when it devastated Luzon. The eyewall of Zeb contracted before landfall and broke down and weakened after landfall; then a much larger new eyewall formed and strengthened as it left Luzon and reentered the ocean. The fifth-generation Pennsylvania State University-NCAR Mesoscale Model (MM5) with four nested domains was used to perform numerical experiments to understand the effects of terrain and land surface variation on the observed eyewall evolution. Results show that the presence of Luzon plays a critical role in the observed eyewall evolution. Quite different from the conventional concentric eyewall replacement, the eyewall replacement that occurred in Typhoon Zeb was triggered by the mesoscale landmass and terrain variation with a horizontal scale similar to the core of the typhoon. In Typhoon Zeb, the original eyewall contracted and broke down because of enhanced surface friction after landfall. The outer eyewall was triggered by convective rainbands near the western coastal region of Luzon and formed as a result of axisymmetrization well after the dissipation of the inner eyewall convection.

Several sensitivity experiments were conducted to elucidate the roles of both condensation heating and planetary boundary layer processes in the evolution of the typhoon eyewall. It is found that although condensational heating is the key to the maintenance of the annular potential vorticity (PV) structure, surface friction plays dual roles. Although friction is a sink to PV and thus dissipates PV in the eyewall, it helps keep the PV annulus narrow by enhancing the stretching deformation in the lower troposphere when condensational heating is present. In the absence of condensational heating, however, surface friction enhances the inward PV mixing by boundary layer frictional inflow and thus destroys the PV annulus.
\end{abstract}

\section{Introduction}

The evolution of the eyewall in tropical cyclones (TCs) has always been an intriguing issue in TC thermodynamics and dynamics (Wang and $\mathrm{Wu} 2004$ ). The variability in the eyewall structure also plays a very important role in TC intensity change. The intensification of a TC is usually accompanied by eyewall contraction, which can be explained easily by the simple

Corresponding author address: Dr. Chun-Chieh Wu, Dept. of Atmospheric Sciences, National Taiwan University, No. 1, Sec. 4, Roosevelt Rd., Taipei 10673, Taiwan.

E-mail: cwu@typhoon.as.ntu.edu.tw
Sawyer-Eliassen model of the response of a balanced vortex to convective heating in the eyewall (Shapiro and Willoughby 1982; Schubert and Hack 1982). When concentric eyewalls are present, the amplification and inward propagation of the outer eyewall usually suppresses and eventually kills the convection in the inner eyewall, leading to an eyewall replacement (Willoughby et al. 1982; Willoughby 1990; Black and Willoughby 1992; Willoughby and Black 1996). A similar eyewall replacement process has been recently identified by Wang (2008a) in the formation of annular hurricanes but with no secondary wind maximum associated with the outer convective ring because of its proximity to the primary eyewall. Recent studies attributed 

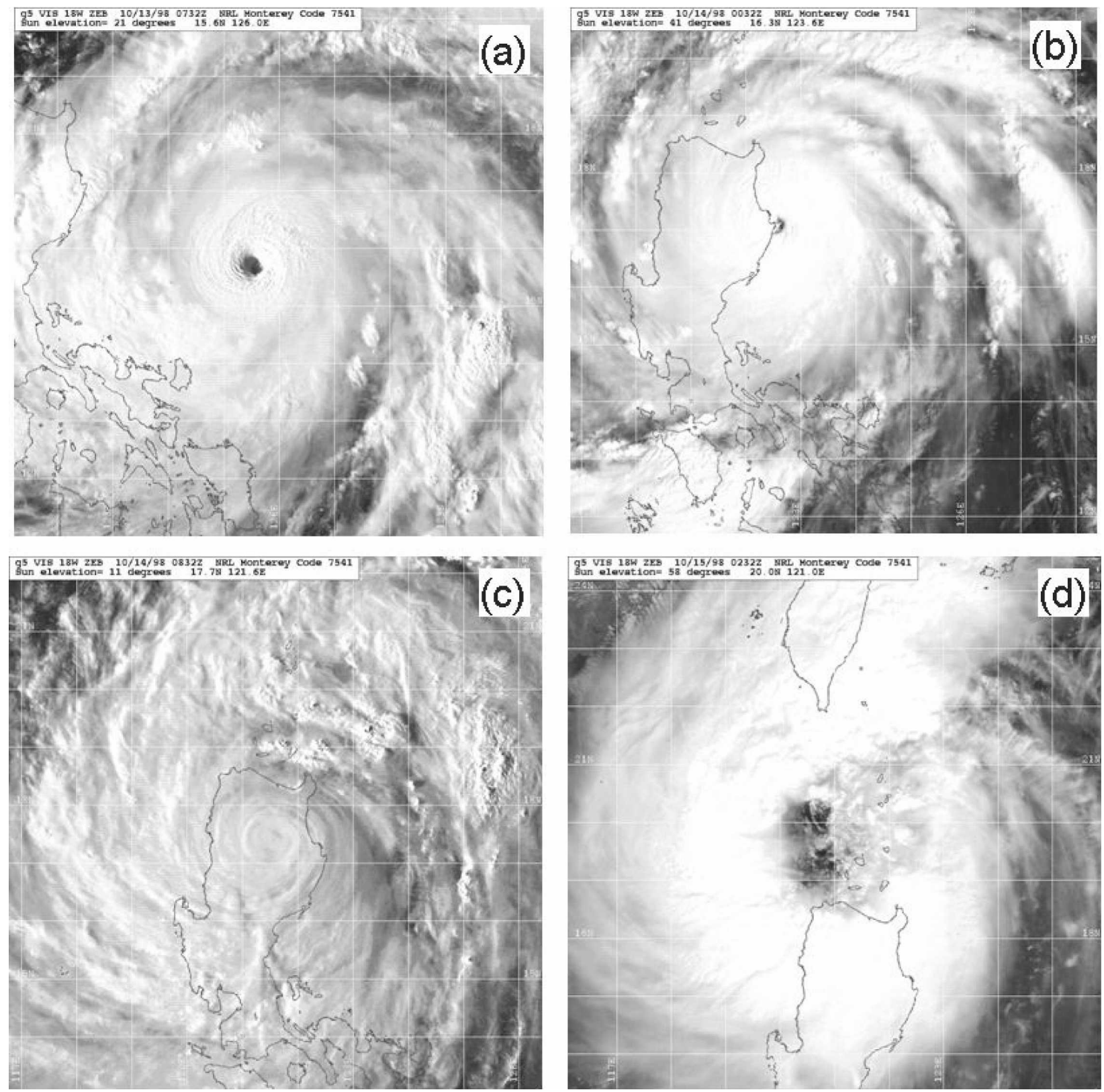

FIG. 1. The Geostationary Meteorological Satellite-5 (GMS-5) visible images for Typhoon Zeb (1998): (a) 0732 UTC 13 Oct; (b) 0032 UTC 14 Oct; (c) 0832 UTC 14 Oct; and (d) 0232 UTC 15 Oct.

polygonal eyewalls and eyewall mesovortices to barotropic instability and the resultant asymmetric mixing processes (Schubert et al. 1999; Reasor et al. 2000). It was also found that the asymmetric vorticity mixing between the eye and eyewall of a TC could lead to pressure falls in the TC core (Schubert et al. 1999; Kossin and Eastin 2001; Kossin and Schubert 2001). Other studies also suggested that the interaction of the vorticity asymmetries and mean vortex could either spin up the mean vortex through the vortex Rossby wave-mean flow interaction (Montgomery and Kallenbach 1997; Montgomery and Enagonio 1998) or limit the maximum intensity of a TC by spinning down the maximum tangential wind through eddy diffusion $(\mathrm{Wu}$ and Braun 2004; Yang et al. 2007). Furthermore, it has been shown in Emanuel (1997) that the maintenance of a narrow annulus of the inner-core eyewall is frontogenetic by nature and would naturally tend toward an infinitely narrow annulus with infinite vorticity (i.e., a circular vortex sheet). 

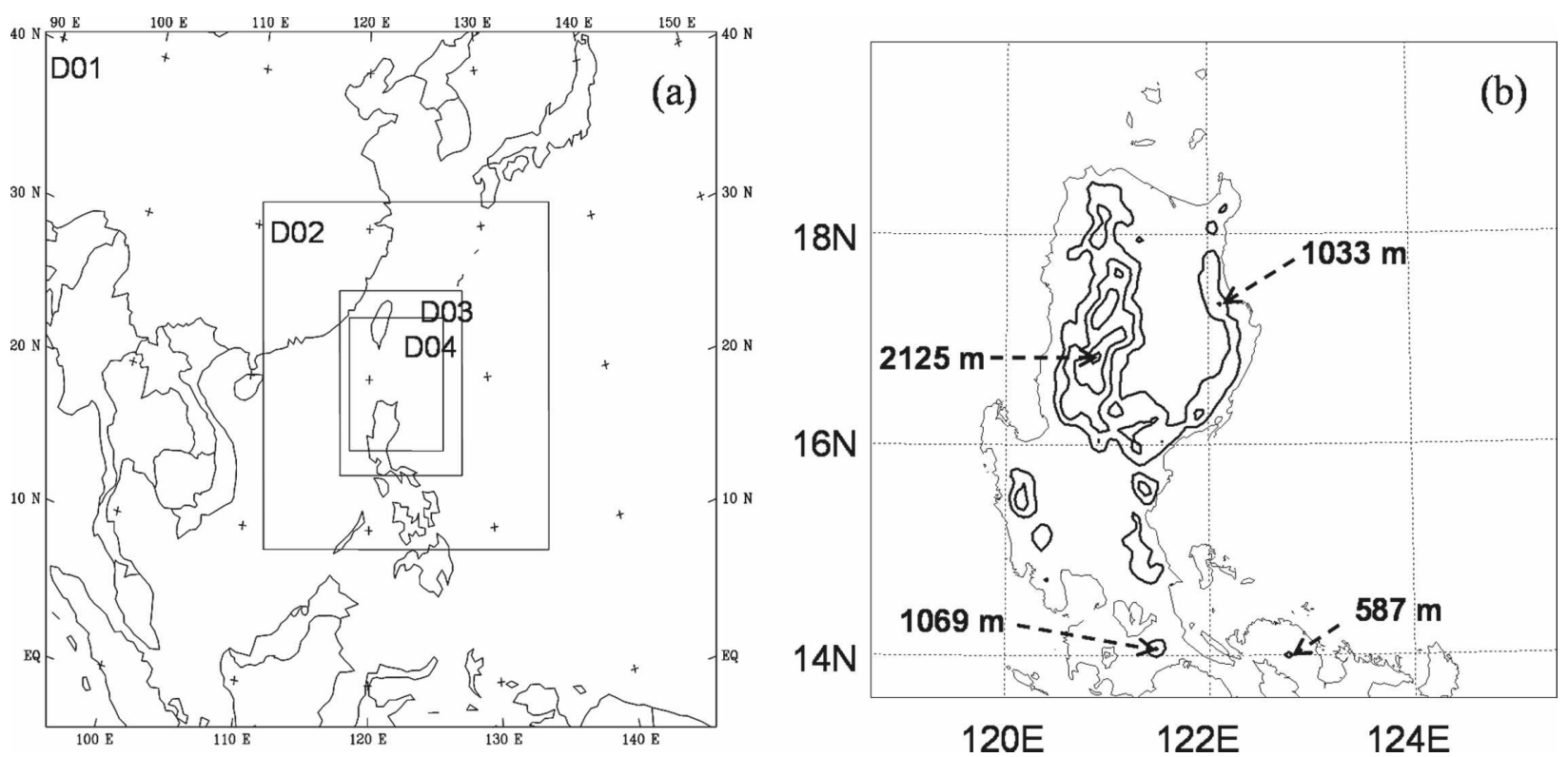

FIG. 2. (a) The four nested domains in the three major experiments. (b) The model's terrain height (contour interval of $500 \mathrm{~m}$ ) of Luzon within the third mesh in CTL.

A number of studies have investigated the evolution of landfalling TCs (Brand and Blelloch 1973, 1974; Yeh and Elsberry 1993a,b; Wu and Kuo 1999; Wu 2001; Wu et al. 2002; Chen and Yau 2003). Whereas most of the above studies focused on investigating the impact of Taiwan terrain, Brand and Blelloch (1973) were the first to document the effect of the Philippine Islands on the changes of track, eye diameter, storm intensity, and size for TCs during 1960-70 based on the annual typhoon reports of the Joint Typhoon Warning Center (with aircraft reconnaissance available during that period). It was indicated that the frictional effect of the landmass and the reduction in heat and moisture supply from the ocean are the primary causes of the above changes, although no specific analyses were conducted to assess the physical mechanisms involved. In general, there have been few studies focusing on the eyewall dynamics of typhoons making landfall on the Philippine Islands, which have terrain features with horizontal scales similar to the size of a TC. Wu et al. (2003a) conducted an observational and modeling study to document the eyewall evolution of Typhoon Zeb (1998) when Zeb interacted with the terrain of Luzon.

As an extension of the study by $\mathrm{Wu}$ et al. (2003a), this study further examines the roles of landmass and the terrain of Luzon in producing the interesting eyewall evolution of Typhoon Zeb (1998) before, during, and after its landfall. As we can see from the satellite images given in Fig. 1, the eyewall of Zeb contracted before landfall and broke down and dissipated after landfall; then a new and much larger eyewall formed as Zeb left Luzon and reentered the ocean. The new eyewall contracted again but remained large as Zeb moved along the east coast of Taiwan. Similar features have also been observed in other TCs, such as Typhoon Melor (2003) over Luzon and Hurricane Wilma (2005) over the Yucatan Peninsula, but these special eyewall evolutions have never been investigated in detail in the literature.

The objectives of this study are twofold: (i) to investigate the effects of terrain, land surface, and ocean on the evolution of the eyewall of Typhoon Zeb and (ii) to understand the dynamics of the eyewall evolution and the associated intensity change of a landfalling typhoon. Several sensitivity experiments are also conducted to explore the effects of moist convection, surface heat flux, and surface friction on the maintenance of the narrow potential vorticity (PV) annulus in the new large eyewall. The experimental design is described in section 2. Results from full-physics simulations and from several sensitivity experiments are discussed in sections 3 and 4, respectively. The major findings are summarized in the last section.

\section{Experimental design}

The numerical simulations are conducted with the fifth-generation Penn State University-National Center for Atmospheric Research (NCAR) Mesoscale Model (MM5; Grell et al. 1995). The nonhydrostatic MM5 
TABLE 1. Summary of all experiments.

\begin{tabular}{llccccc}
\hline \hline $\begin{array}{c}\text { Expt } \\
\text { name }\end{array}$ & Luzon terrain & Moisture & $\begin{array}{c}\text { Surface } \\
\text { heat flux }\end{array}$ & $\begin{array}{c}\text { Surface } \\
\text { friction }\end{array}$ & $\begin{array}{c}\text { Simulation } \\
\text { period }\end{array}$ & Eyewall evolution during 54-72h \\
\hline CTL & Full & Yes & Yes & Yes & $0-72 \mathrm{~h}$ & $($ MSLP $=962-954 \mathrm{hPa}) \mathrm{PV}$ ring \\
NLT & Flat & Yes & Yes & Yes & $0-72 \mathrm{~h}$ & \\
SEA & Virtual ocean & Yes & Yes & Yes & $0-72 \mathrm{~h}$ & \\
DFL & Full & No & No & No & $54-72 \mathrm{~h}$ & $($ MSLP $=962-971 \mathrm{hPa})$ Mixing and dissipating \\
DRY & Full & No & Yes & Yes & $54-72 \mathrm{~h}$ & $($ MSLP $=962-991 \mathrm{hPa})$ Rapid dissipating \\
NHF & Full & Yes & No & Yes & $54-72 \mathrm{~h}$ & $($ MSLP $=962-973 \mathrm{hPa})$ Rapid mixing \\
FL & Full & Yes & No & No & $54-72 \mathrm{~h}$ & $($ MSLP $=962-952 \mathrm{hPa})$ Distorted PV ring with mesovortices \\
\hline
\end{tabular}

with four nested domains (Fig. 2a) is used to perform 72-h simulations, starting from 0000 UTC 13 October 1998. The model was run with 23 vertical levels $(0.025$, $0.075,0.125,0.175,0.225,0.275,0.325,0.375,0.425$, $0.475,0.525,0.575,0.625,0.675,0.725,0.775,0.825,0.87$, $0.91,0.945,0.97,0.985$, and 0.995$)$ in the terrainfollowing $\sigma$ coordinate $\left[\sigma=\left(p-p_{t}\right) /\left(p_{s}-p_{t}\right)\right.$, where $p$ is the pressure, $p_{s}$ the surface pressure, and $p_{t}$ the constant top pressure of $10 \mathrm{hPa}$. The horizontal grid spacings of the four domains are $54 \mathrm{~km}$ (the first mesh, $95 \times$ 109 grid points), $18 \mathrm{~km}$ (the second mesh, $142 \times 139$ grid points), $6 \mathrm{~km}$ (the third mesh, $226 \times 178$ grid points), and $2 \mathrm{~km}$ (the fourth mesh, $487 \times 409$ grid points). The initial and lateral boundary conditions are based on the European Centre for Medium-Range Weather Forecasts (ECMWF) advanced global analysis. The model initialization and vortex bogusing are based on the method described in Wu et al. (2002). The planetary boundary layer (PBL) scheme used in the model is the Blackadar formula (Blackadar 1976, 1979;
Zhang and Anthes 1982). In the two coarser meshes (with 54- and 18-km horizontal grid spacings), cumulus convection is parameterized with the Betts-Miller scheme (Betts and Miller 1986). The explicit cloud microphysics scheme of Reisner et al. (1998) is used for all meshes.

Three full-physics numerical experiments with different underlying surface conditions are conducted to investigate the effects of the terrain of Luzon, the land surface, and the ocean on the evolution of the eyewall of Zeb. The control experiment (CTL; see Table 1 for descriptions of all experiments) retains all the modelresolved terrain in the model domain. In the second experiment (NLT), the mountains of Luzon (with a maximum height of $2125 \mathrm{~m}$ in the third mesh; Fig. 2b) are flattened. In the third experiment (SEA), the land of Luzon is totally replaced with the ocean, where the "sea" surface temperature over Luzon is interpolated from the boundary value around the replaced area. To save computing time, each of the three simulations is
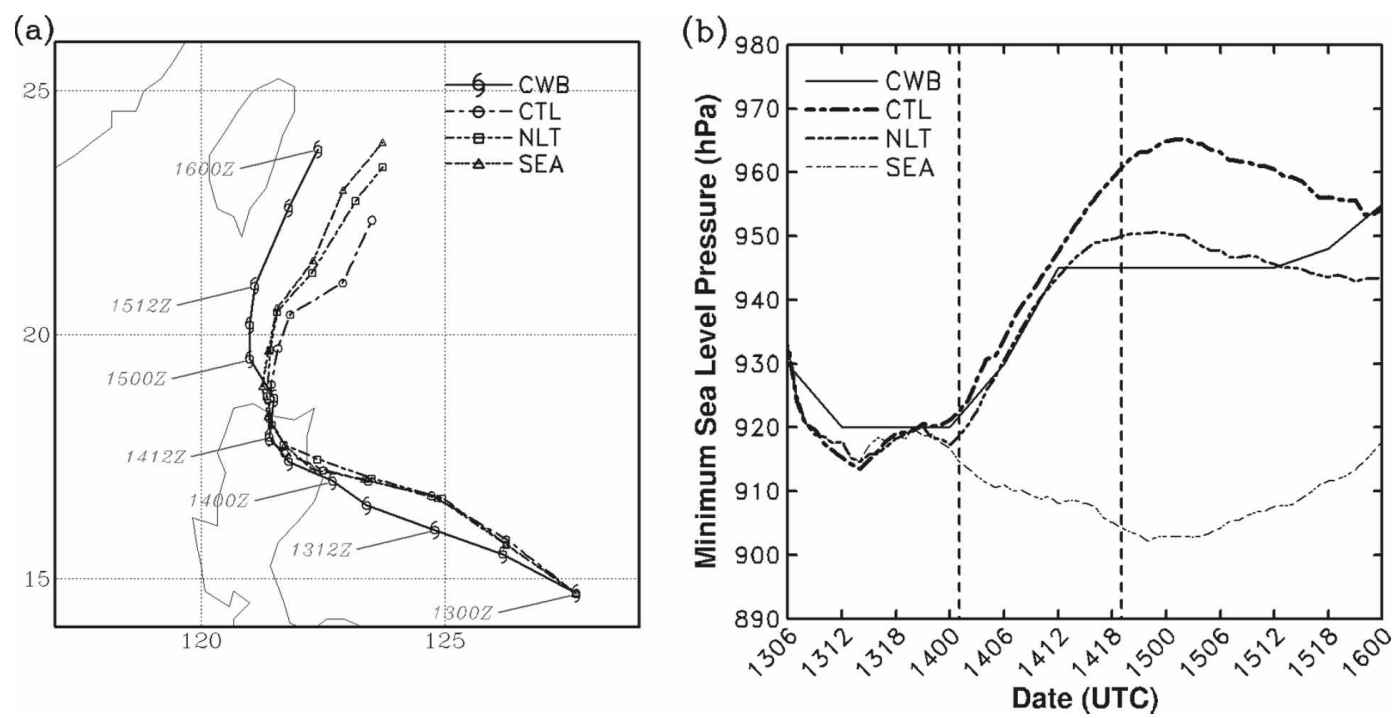

FIG. 3. (a) Best track of CWB and model tracks of all experiments; (b) minimum sea level pressure of Typhoon Zeb. The left (right) vertical dashed line indicates the time when Zeb made landfall at (exited from) Luzon. 

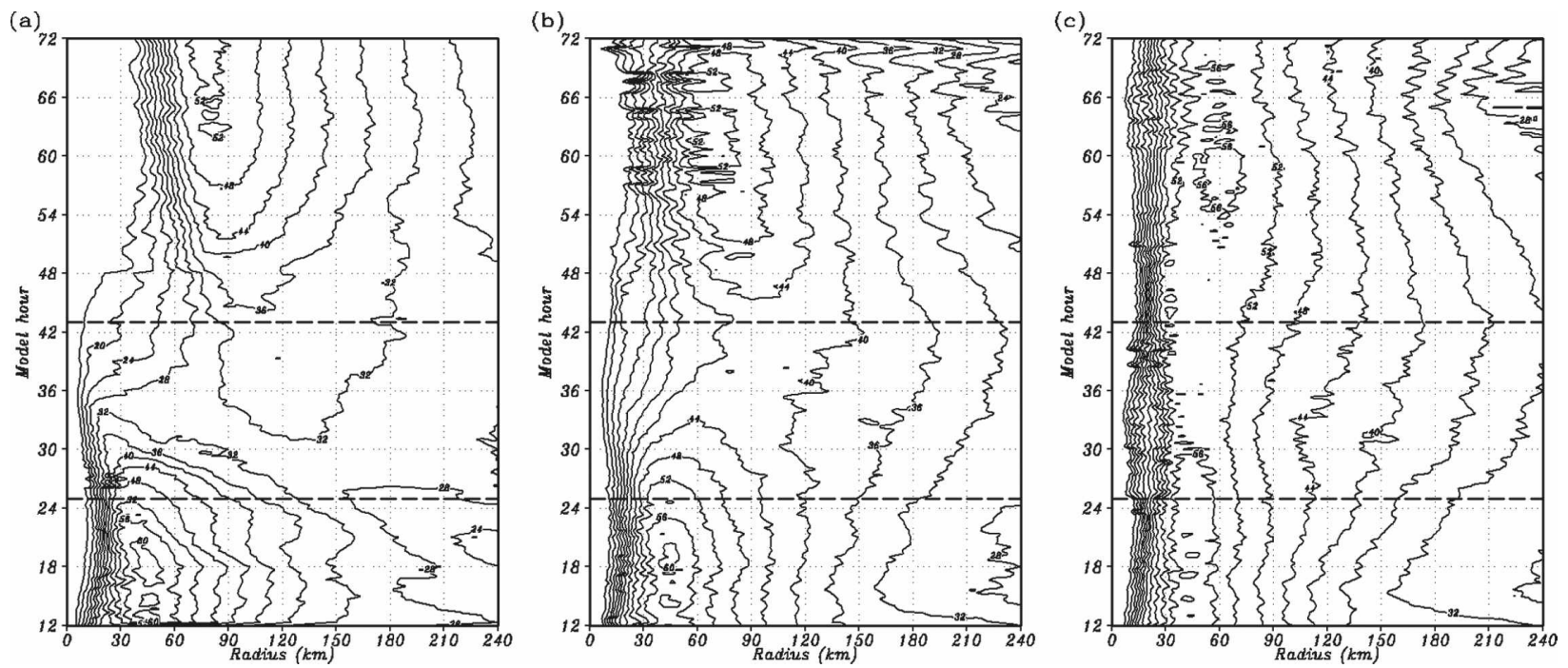

FIG. 4. Radius-time Hovmöller diagram of azimuthally averaged tangential wind $\left(\mathrm{m} \mathrm{s}^{-1}\right)$ at $925 \mathrm{hPa}$ for (a) CTL, (b) NLT, and (c) SEA. The lower (upper) horizontal long-dashed line indicates the time when Zeb made landfall at (exited from) Luzon.

(a)

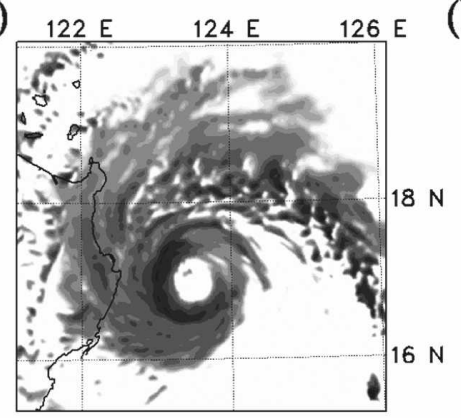

(d)

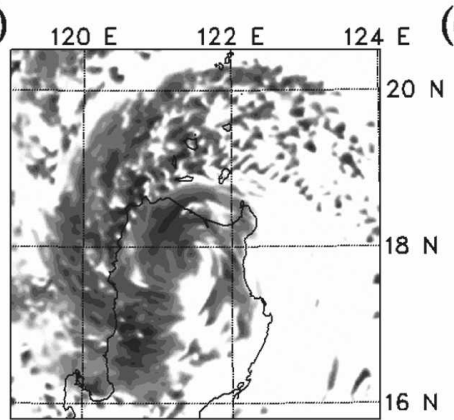

(g)

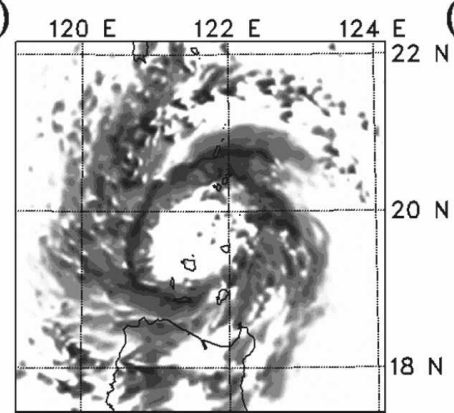

(b)

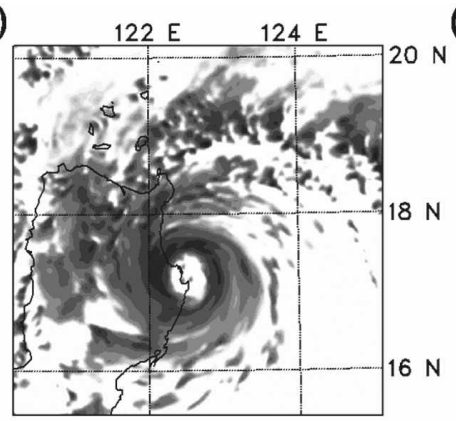

(e)

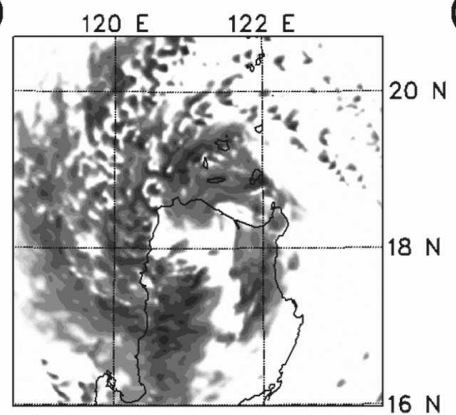

(h)

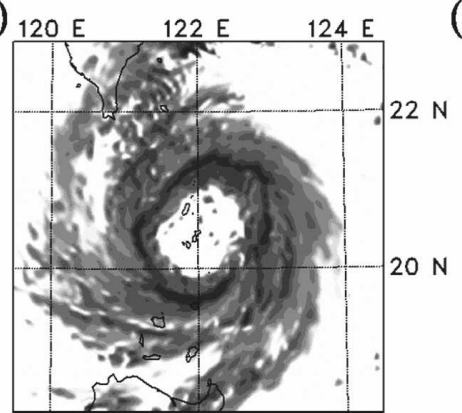

(c)

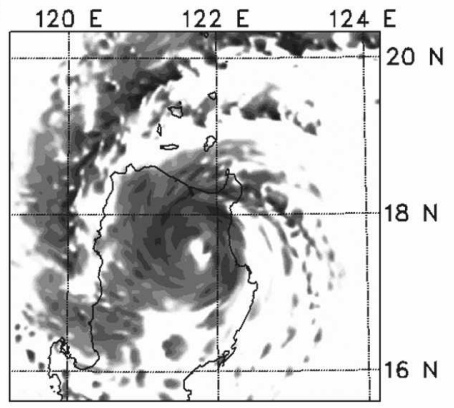

(f)

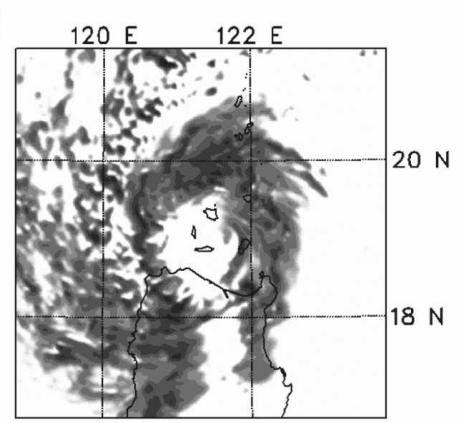

(i)

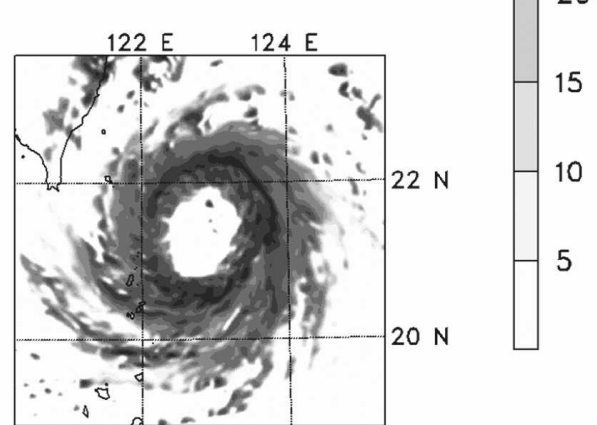

FIG. 5. The low-level $(\sigma=0.91)$ radar reflectivity (dBZ) in CTL at (a) 18, (b) 24, (c) 30, (d) 36, (e) 42, (f) 48, (g) 54, (h) 60, and (i) 66 h. 
(a)

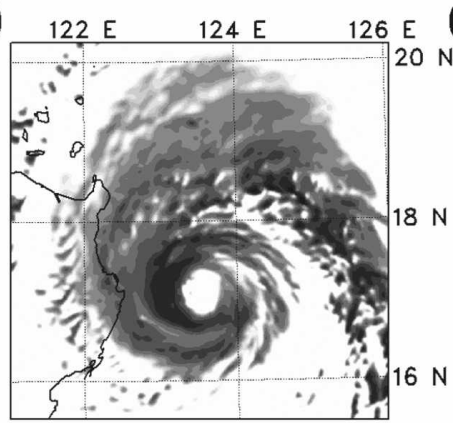

(d)

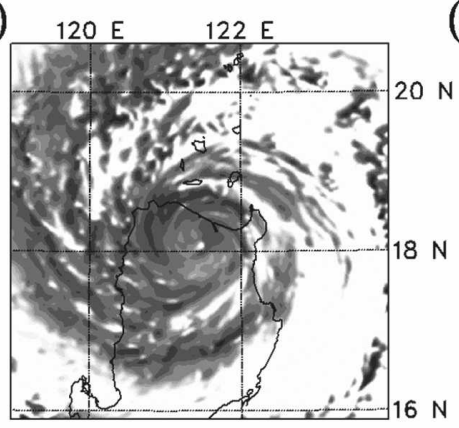

(g)

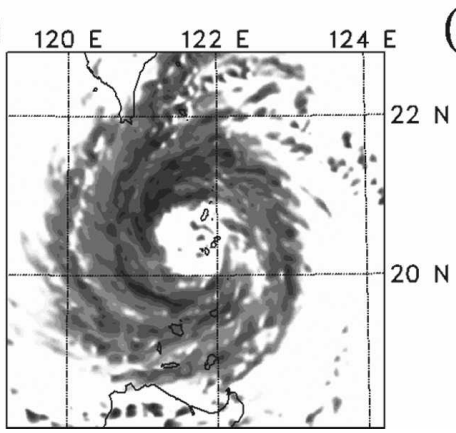

(b)

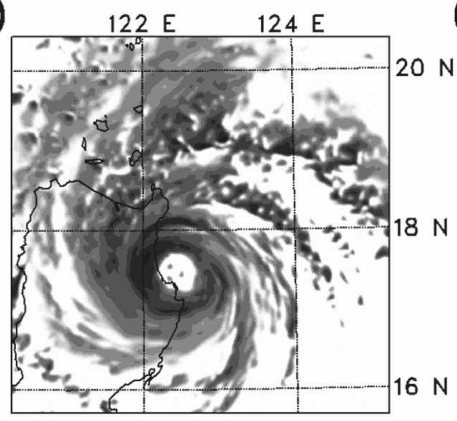

(e)

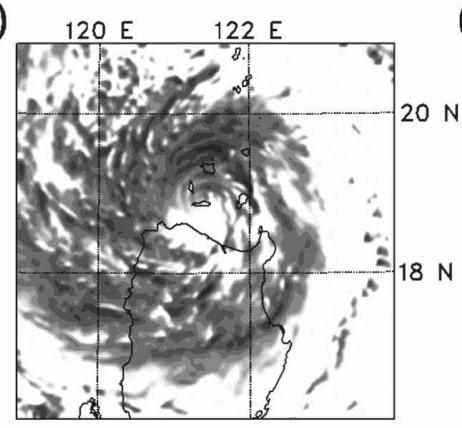

(h)

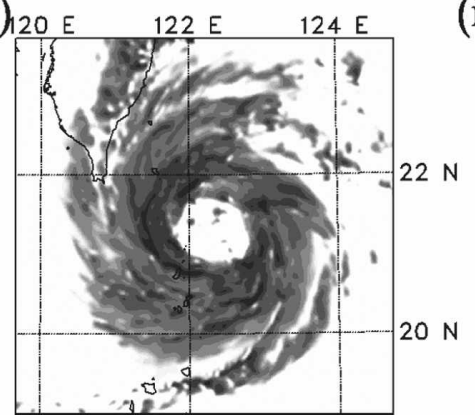

(c)

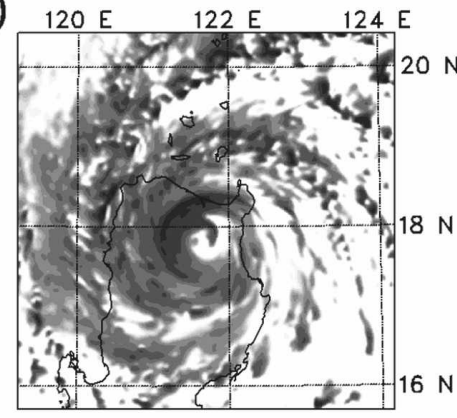

(f)

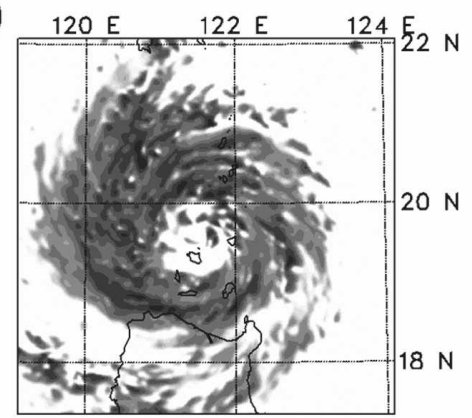

(i)

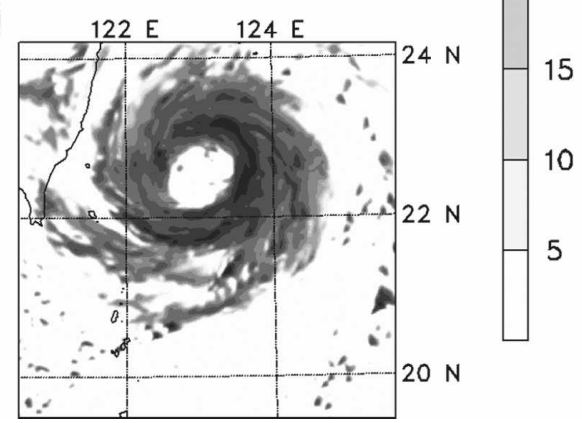

FIG. 6. As in Fig. 5, but for NLT.

first performed with the two outer meshes only. The simulation on the third mesh is started at $t=4 \mathrm{~h}$. The initial field of the third mesh, except for the terrain height, is interpolated from the output of the second mesh at $t=4 \mathrm{~h}$ as well. At $t=12 \mathrm{~h}$, the fourth mesh is activated with the initial field interpolated from the output of the third mesh. Most of the results shown in the following sections are from the fourth domain. Results from these full-physics simulations are discussed in section 3 .

To further understand the physical processes responsible for the maintenance of the eyewall PV annulus of the simulated Zeb, we have performed four sensitivity experiments with varying model physics (see Table 1). All sensitivity experiments are initialized with the output of the CTL experiment at $54 \mathrm{~h}$ and integrated for 18 $\mathrm{h}$ with the lateral boundary conditions from the 54-72-h output of the CTL experiment. In the first sensitivity experiment (DFL), the MM5 is integrated with neither moist processes nor the PBL scheme; that is, the experiment is dry, frictionless, and without the surface sensible and latent heat fluxes. In the second sensitivity experiment (DRY), the moist processes are turned off but the PBL scheme is retained. The third sensitivity experiment (NHF) is similar to the CTL experiment but with the surface heat fluxes turned off. The final sensitivity experiment (FL) is the same as CTL but without the PBL scheme. Results from these sensitivity experiments are discussed in section 4 . Note that some level of imbalance is introduced in these sensitivity experiments because of the inconsistencies in the model physics with both the initial and lateral boundary conditions. Nevertheless, these imbalance and inconsistencies are expected not to overtake the actual physics that we intend to explore, although the results should be explained with caution. 

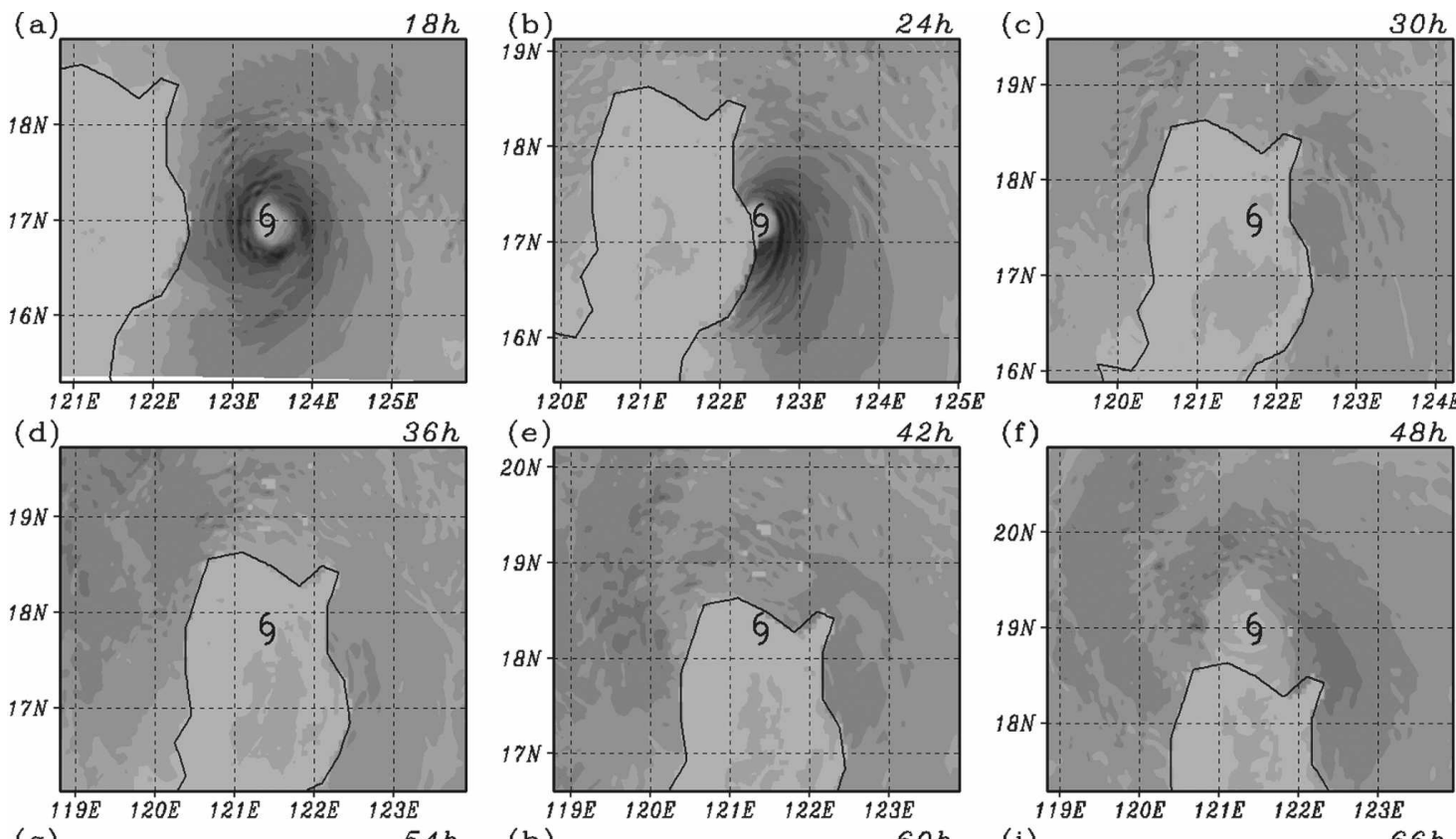

1800
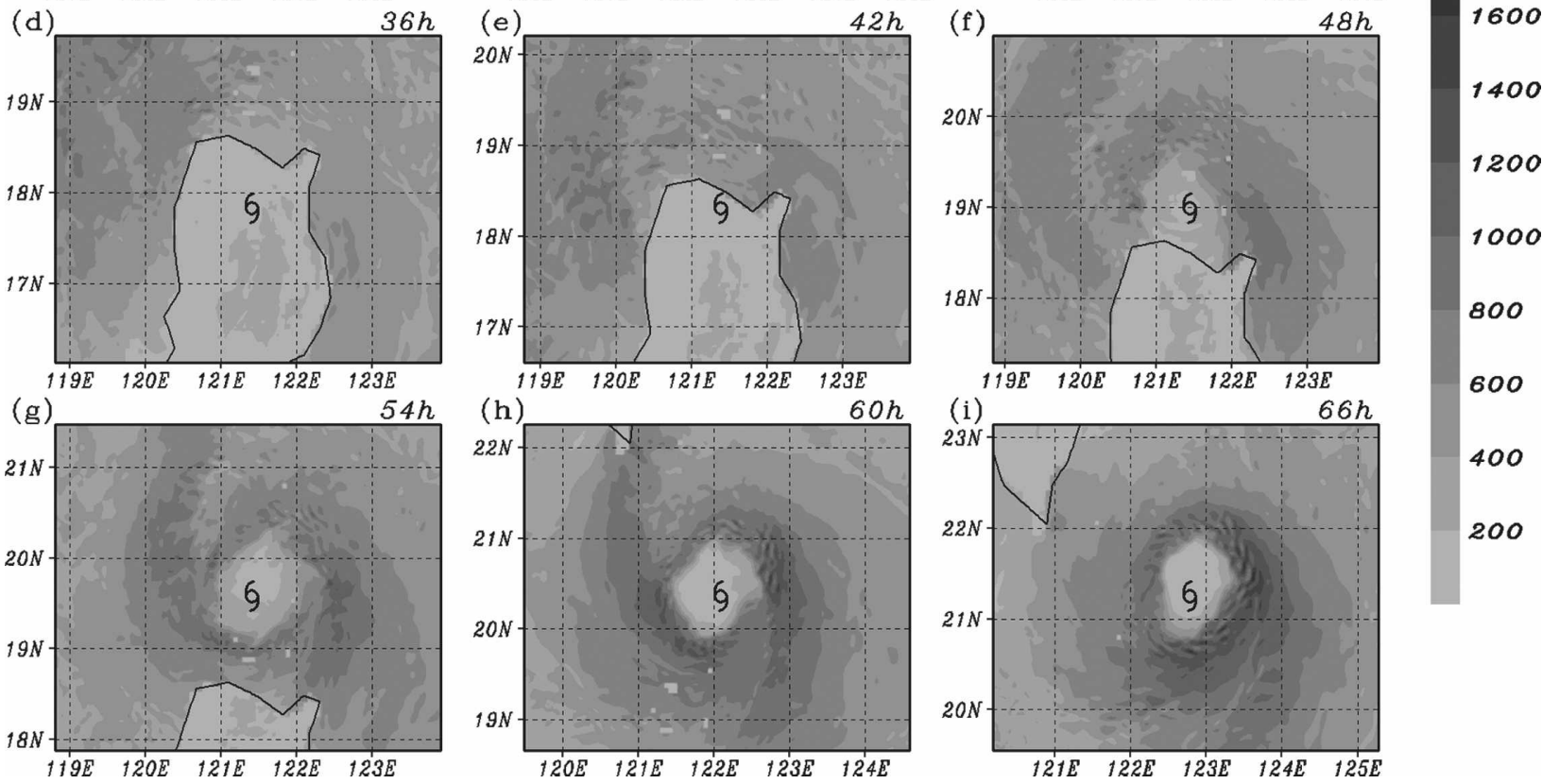

FIG. 7. Surface latent heat flux $\left(\mathrm{W} \mathrm{m}^{-2}\right.$ ) in CTL at (a) 18, (b) 24, (c) 30, (d) 36, (e) 42, (f) 48, (g) 54, (h) 60, and (i) 66 h. The typhoon symbol indicates the location of MSLP.

\section{The effects of terrain and land surface of Luzon}

Except for some slight northward deflection of Zeb's track in the initial $24 \mathrm{~h}$ and an eastward deflection after $48 \mathrm{~h}$, the simulated tracks (the location of the storm is defined as the center of the minimum sea level pressure here $)^{1}$ of all the three full-physics experiments (Fig. 3a) are in general agreement with the best-track analysis of Zeb from the Central Weather Bureau (CWB) of Taiwan. The evolution of the minimum sea level pressure (MSLP) is also well simulated in CTL (Fig. 3b). NLT shows a MSLP evolution similar to that in CTL, especially the filling after landfall at Luzon. The similar filling rates of MSLP in both NLT and CTL, as com-

\footnotetext{
${ }^{1}$ The typhoon center in the later discussion is defined as the PV-weighted center, leading to a more accurate circulation center, especially after the formation of the large eye.
}

pared to the continuous strengthening of the storm in SEA, suggest that the weakening of Zeb after landfall is mainly due to the great reduction of heat fluxes from the underlying surface. Note that the storm intensity after landfall in CTL, although weaker than observed, is generally about $10-15 \mathrm{hPa}$ weaker than that in NLT, indicating that the terrain over Luzon plays a role in weakening the storm. We find that in the presence of terrain, convection outside the eyewall is triggered earlier after the landfall of Zeb. This results in strong suppression and rapid dissipation of the eyewall convection, and thus the weakening of the storm.

The radius-time Hovmöller diagrams of the azimuthally averaged tangential wind from the three fullphysics experiments (Fig. 4) indicate that the breakdown and reformation of the eyewall of Zeb are closely related to the influence of the different underlying surfaces. The main features of the eyewall contraction, breakdown, and reformation processes during the pe- 

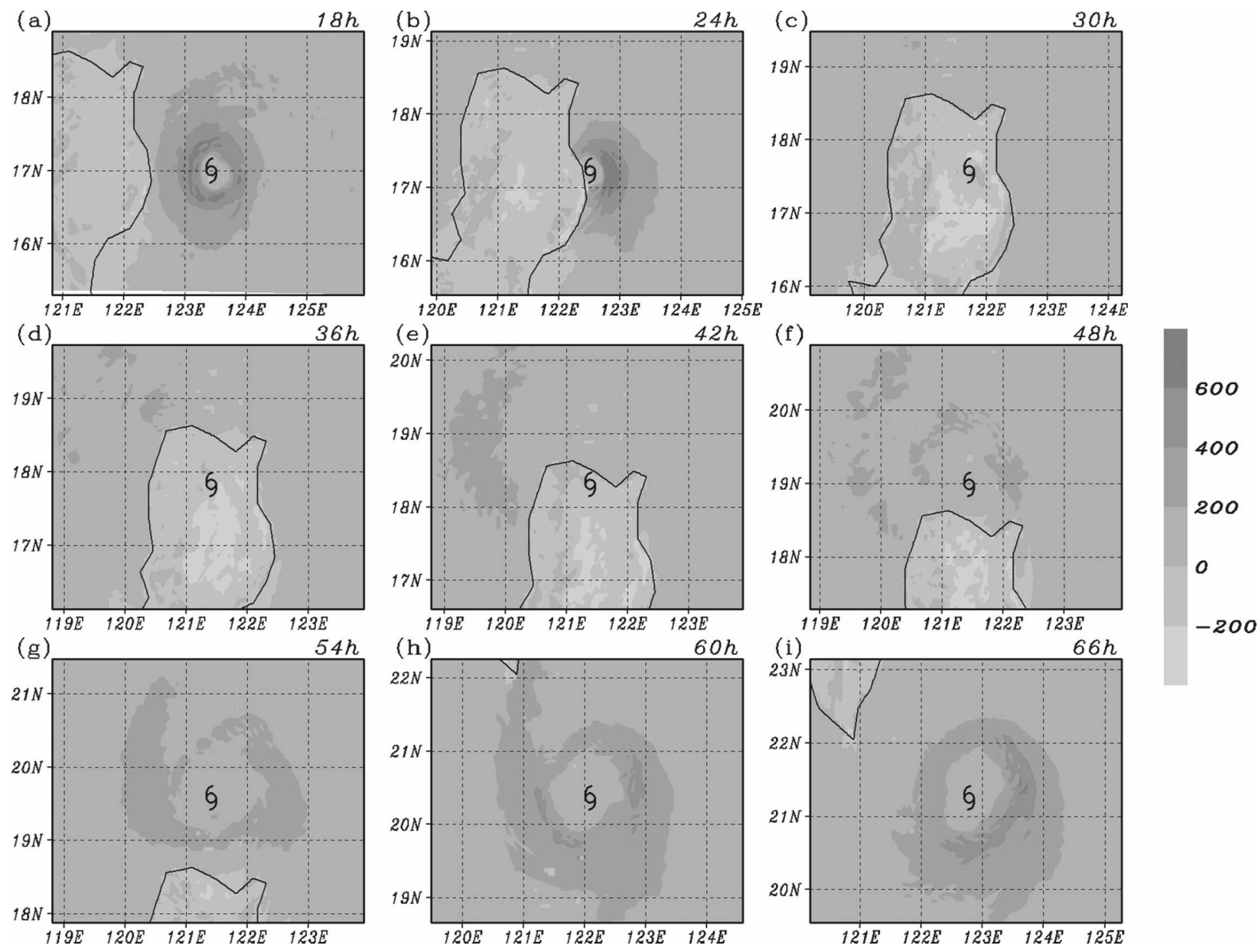

FIG. 8. As in Fig. 7, but for surface sensible heat flux.

riod when Zeb is near Luzon are well captured in CTL. The eyewall contracts before landfall and breaks down and dissipates over land, and a large, new eyewall forms as Zeb leaves Luzon and reenters the ocean. The contraction of the eyewall before landfall is likely enhanced by the orographic effect of Luzon because the contraction is not significant in NLT (i.e., without terrain). Despite a slight decrease of maximum wind between about 20 and $40 \mathrm{~h}$, the simulated storm in SEA intensifies from the beginning of the integration until 50 h (Fig. 3) with nearly constant eyewall size (Fig. 4c).

The evolution of the simulated radar reflectivity at a low model level $(\sigma=0.91$; Fig. 5$)$ in CTL shows a remarkable weakening of convection in the eyewall after the landfall of Zeb. In the first few hours after landfall, the radius of maximum azimuthal mean tangential wind (RMTW) in the boundary layer decreases as the storm weakens. The RMTW at $925 \mathrm{hPa}$ reduces from
$40 \mathrm{~km}$ at $25 \mathrm{~h}$ (when Zeb makes landfall) to $18 \mathrm{~km}$ at $33 \mathrm{~h}$ (Fig. 4). The eyewall convection continues to weaken as the storm moves inland, whereas the cyclonic circulation beyond a radius of $150 \mathrm{~km}$ strengthens (Fig. 4) with the radius of the wind speed $>15 \mathrm{~m} \mathrm{~s}^{-1}$ increasing. It is not until the eyewall dissipates that an outer convective rainband is organized to form a circular convective ring outside the original eyewall. A secondary wind maximum associated with the outer convective ring occurs at a radius of about $120 \mathrm{~km}$ at $32 \mathrm{~h}$. The original eyewall breaks down about $12 \mathrm{~h}$ after landfall. As the original eyewall convection continues to weaken and the maximum wind decreases, the outer convective ring strengthens and the secondary maximum wind increases. The outer eyewall develops more rapidly after the storm reenters the ocean, and the storm then continues to reintensify. The convection in the original eyewall decays and a larger new eyewall appears at around $52 \mathrm{~h}$ (figure not shown). Our results indicate that the for- 

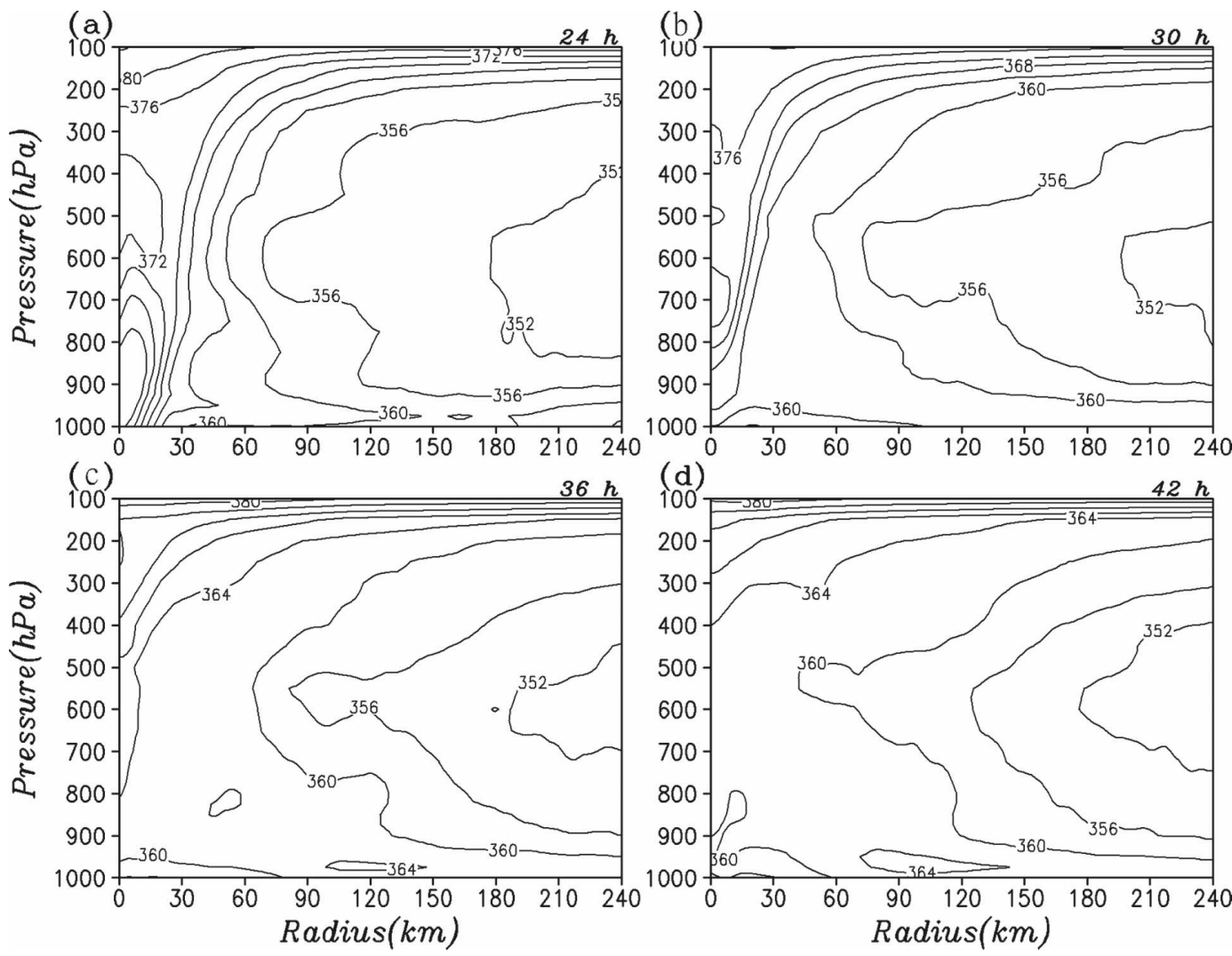

FIG. 9. The azimuthal mean equivalent potential temperature (contour interval of $4 \mathrm{~K}$ ) profile in CTL at (a) 24, (b) 30, (c) 36, and (d) $42 \mathrm{~h}$.

mation of the larger new eyewall of Zeb is due to the weakening and dissipation of the original eyewall and the development of the outer eyewall.

With the terrain removed in NLT, the eyewall does not contract before landfall (Fig. 4b), although its breakdown and reformation processes resemble those in CTL. In NLT, the maximum tangential wind decreases after landfall, with the RMTW increasing at the same time (Fig. 4b), in contrast to the RMTW decrease in CTL. However, similar to what happens in CTL, a larger, new eyewall appears several hours after the storm reenters the ocean (Fig. 6), but the radius of the new eyewall in NLT is smaller than that in CTL.

The different eyewall evolution in the three fullphysics experiments indicates that the interesting eyewall evolution of Zeb is closely associated with the landfall of Zeb and its interaction with the terrain of Luzon. Moreover, the radius of the eyewall increases gradually in NLT and more abruptly in CTL. The formation of the outer eyewall in both CTL and NLT is associated with the development and organization of the outer spiral rainbands. The mountains of Luzon play an important role in enhancing the convection in the outer rainbands such that the new eyewall forms more rapidly in CTL than in NLT.

The distribution of the surface sensible and latent heat fluxes shows that the maximum surface heat fluxes are located underneath the eyewall before Zeb makes landfall (Figs. 7 and 8). The surface heat fluxes under the eyewall decrease remarkably as Zeb moves inland. The decrease of the low-level equivalent potential temperature (Fig. 9) indicates that the air in the PBL in the eye cools and dries significantly after Zeb makes landfall. As a result, the convection in the eyewall is greatly suppressed. On the other hand, although the surface sensible and latent heat fluxes from the underlying surface under the eyewall are reduced, those outside the core remain large because of the maintenance of the outer circulation of the storm. Moreover, in CTL, the low-level convergence along the western coast is enhanced by the mountain of Luzon initiating and/or enhancing convective rainbands to the west coast of $\mathrm{Lu}-$ zon (Fig. 5). The rainbands are later axisymmetrized to form an outer convective ring. In such an eyewall evolution process, the dissipation of the original eyewall that occurs prior to the formation of the new outer 

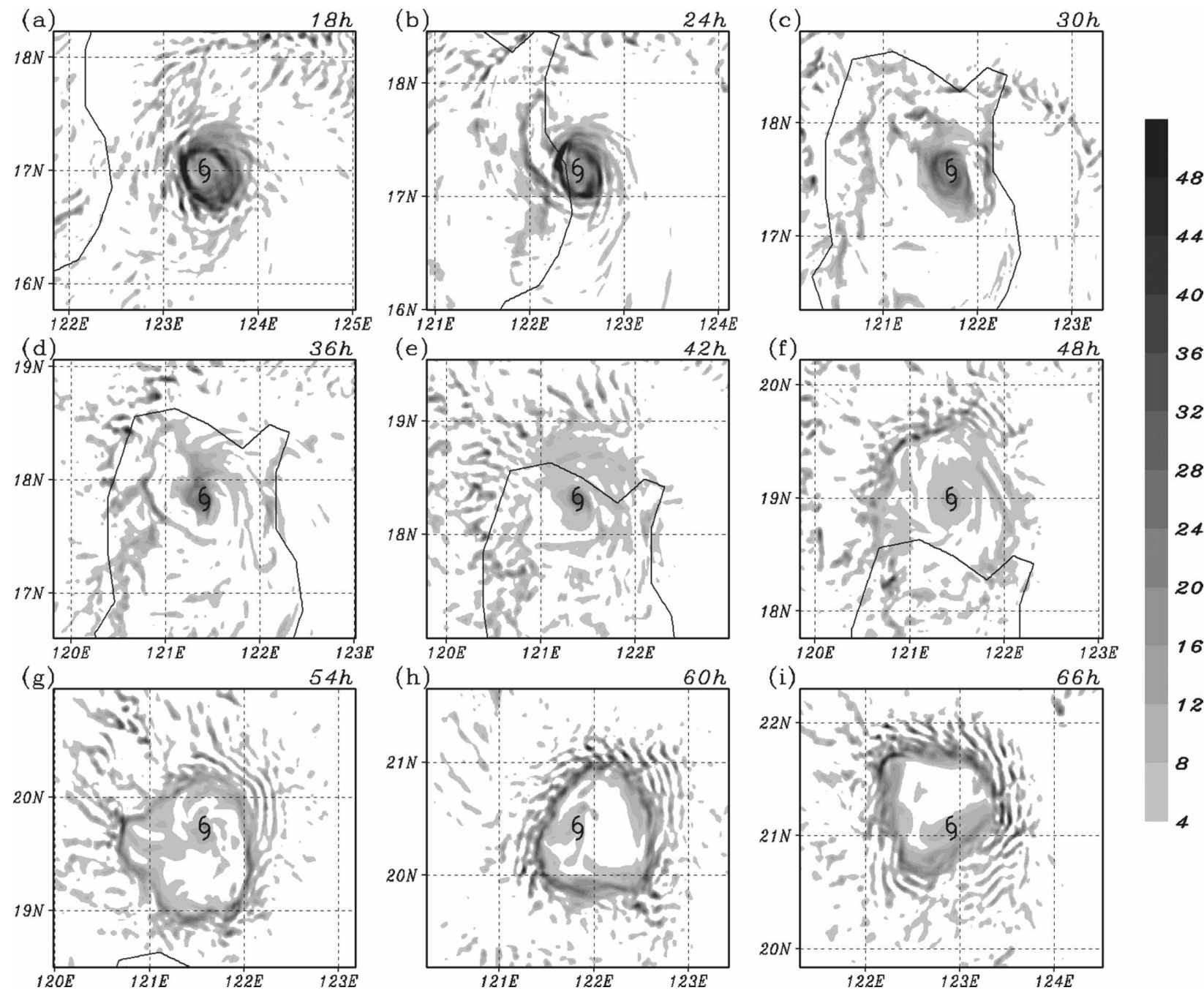

Fig. 10. The PV (PVU; 1 PVU $=1 \times 10^{-6} \mathrm{~m}^{2} \mathrm{~s}^{-1} \mathrm{~K} \mathrm{~kg}^{-1}$ ) at $875 \mathrm{hPa}$ in CTL at (a) 18, (b) 24, (c) 30, (d) 36, (e) 42, (f) 48, (g) 54, (h) 60, and (i) $66 \mathrm{~h}$. The typhoon symbol indicates the location of MSLP.

eyewall is triggered by the landfall, whereas the availability of the surface sensible and latent heat fluxes plays a critical role in the formation of the new large eyewall.

PV is a quantity useful for understanding many aspects of inner core dynamics of TCs (e.g., Wang 2002a,b). Ertel's PV is approximated (ignoring the horizontal gradient of the vertical velocity in the definition of horizontal vorticity; Wu et al. 2003b, 2004) as

$$
q=-\frac{g \kappa \pi}{p}\left(\eta \frac{\partial \theta}{\partial \pi}-\frac{1}{a \cos \varphi} \frac{\partial v}{\partial \pi} \frac{\partial \theta}{\partial \lambda}+\frac{1}{a} \frac{\partial u}{\partial \pi} \frac{\partial \theta}{\partial \varphi}\right)
$$

where $\kappa=R_{d} / C_{p} ; u$ and $v$ are the storm-relative radial and tangential winds, respectively; $\eta$ denotes the vertical component of the absolute vorticity; the vertical coordinate $\pi$ is the Exner function $\left[\pi=C_{p}\left(p / p_{o}\right)^{\kappa}\right]$; and $\theta$ and $g$ denote the potential temperature and gravitational acceleration, respectively.

The PV evolution in the two landfall experiments suggests that the increased surface friction and the reduced surface sensible and latent heat fluxes could both disrupt the maintenance of the annular PV structure of the eyewall over land. As shown in Figs. 10 and 11, before Zeb makes landfall, the maximum PV is located just inside the RMTW. The small high-PV annulus collapses (Fig. 10a) after Zeb makes landfall. The PV annulus evolves into a monopole in the $12 \mathrm{~h}$ after landfall (Figs. 10d and 11). Similar evolution occurs in NLT (Fig. 12). In the two landfall experiments, the low-level PV evolution after Zeb makes landfall resembles the PV mixing between the eye and the eyewall described 


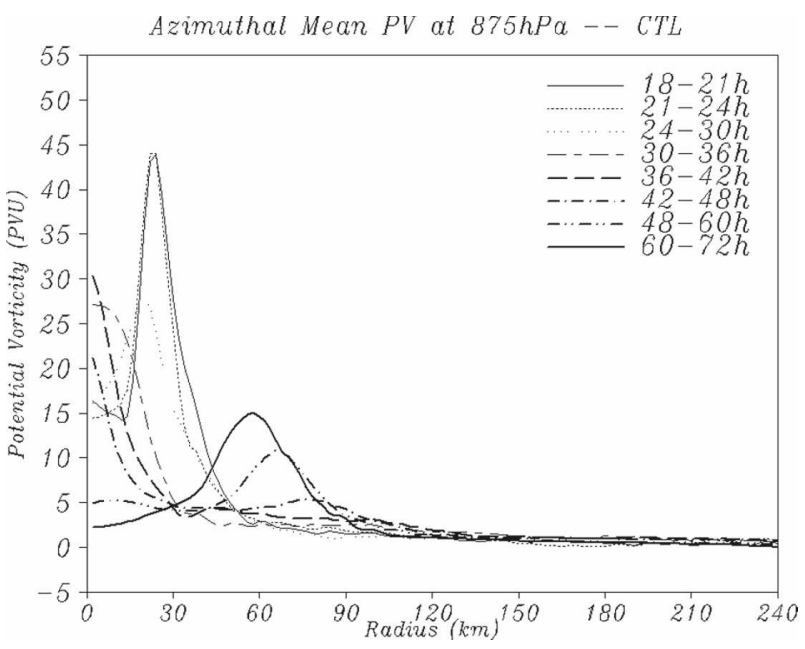

FIG. 11. The azimuthal mean PV (PVU) at $875 \mathrm{hPa}$ at different stages in CTL. in the observational study of Kossin and Eastin (2001) and the theoretical study of Schubert et al. (1999) based on an unforced barotropic model. However, in our case, it seems that either the reduced moist convection or the increased surface friction or both after landfall could lead to the enhanced PV mixing between the eye and the eyewall. The annular nature of the eyewall PV is easily maintained over the ocean, whereas a monopolar PV structure dominates over land. The relative roles of moist processes and surface friction in the maintenance of the large outer PV annulus will be further discussed in the next section through several sensitivity experiments with varying model physics.

In CTL, the new large PV annulus associated with the outer eyewall forms before Zeb reenters the ocean and becomes well organized after Zeb reenters the ocean (Figs. 10e,f). When the new outer eyewall is en-
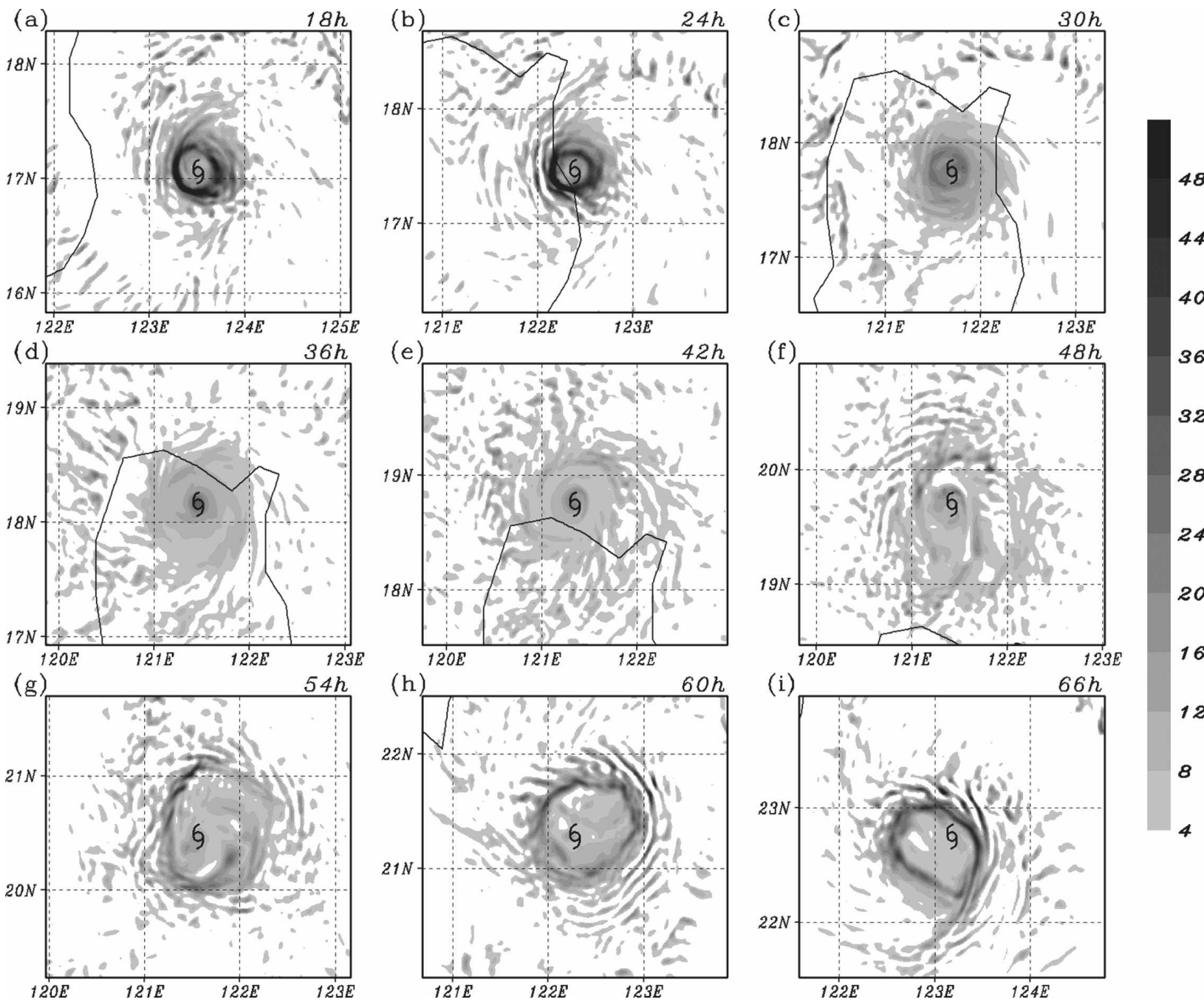

FIG. 12. As in Fig. 10, but for NLT. 

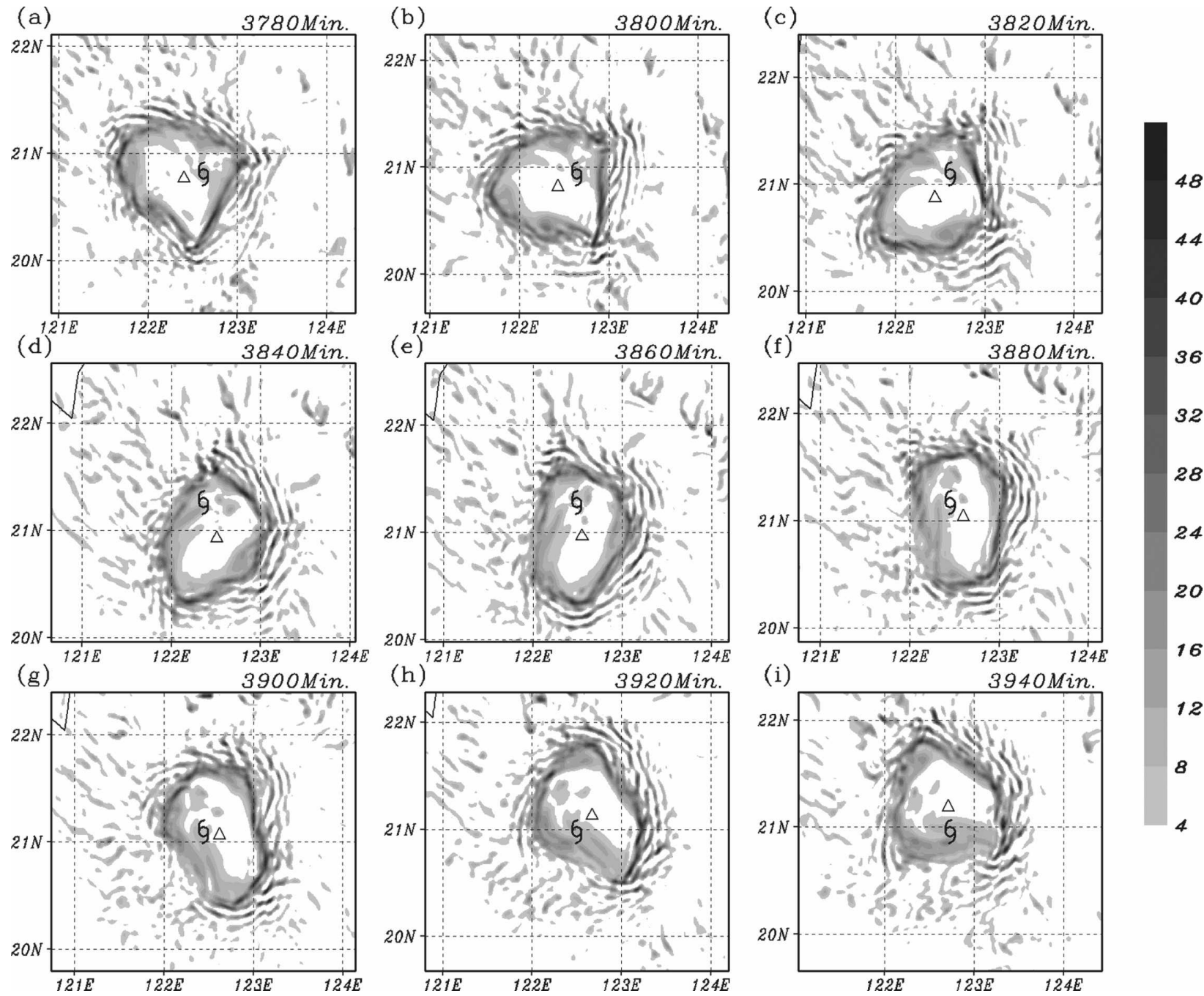

Fig. 13. The PV (PVU) at $875 \mathrm{hPa}$ in CTL at (a) $63 \mathrm{~h}$, (b) $63 \mathrm{~h} 20 \mathrm{~min}$, (c) $63 \mathrm{~h} 40 \mathrm{~min}$, (d) $64 \mathrm{~h}$, (e) $64 \mathrm{~h} 20 \mathrm{~min}$, (f) $64 \mathrm{~h} 40 \mathrm{~min}$, (g) $65 \mathrm{~h}$, (h) $65 \mathrm{~h} 20 \mathrm{~min}$, and (i) $65 \mathrm{~h} 40 \mathrm{~min}$. The typhoon symbol indicates the location of MSLP; the triangle indicates the low-level PV-weighted center.

tirely over the ocean, the high-PV annulus exhibits a distinct wavelike asymmetric structure, evolving into different polygonal patterns (Fig. 13). The polygonal patterns, as well as the location of the MSLP (shown with a typhoon symbol), rotate cyclonically around the low-level PV-weighted center (shown with a triangle). At the same time, the central monopolar PV patch weakens and migrates toward the outer PV annulus (Fig. 12h) and then orbits along the inner edge of the outer high-PV annulus (Fig. 13). Eventually, the monopolar PV patch is distorted and merges into the new eyewall PV annulus.

To further examine the mechanism of the outer eyewall development, PV budget analyses are carried out. The local PV change,

$$
\begin{aligned}
\frac{\partial q}{\partial t}= & -v_{h} \cdot \nabla q-\omega^{*} \frac{\partial q}{\partial \pi} \\
& -\frac{g \kappa \pi}{p}\left[\eta \cdot \nabla\left(\frac{d \theta}{d t}\right)+\nabla \theta \cdot \nabla \times F\right]
\end{aligned}
$$

(as in Wu and Kurihara 1996), is governed by the horizontal and vertical advection of PV (where $\omega^{*}=d \pi / d t$ ), diabatic heating $(d \theta / d t$, including the condensational and radiative heating), and friction.

The PV budget analyses at $700 \mathrm{hPa}$ at $38 \mathrm{~h}$ (Fig. 14) indicate that diabatic (condensation) heating contributes to the production of high PV in the outer eyewall, especially in the lower levels (figures not shown). The redistribution of the high PV is accomplished through the cyclonic advection by the swirling flow of the storm 


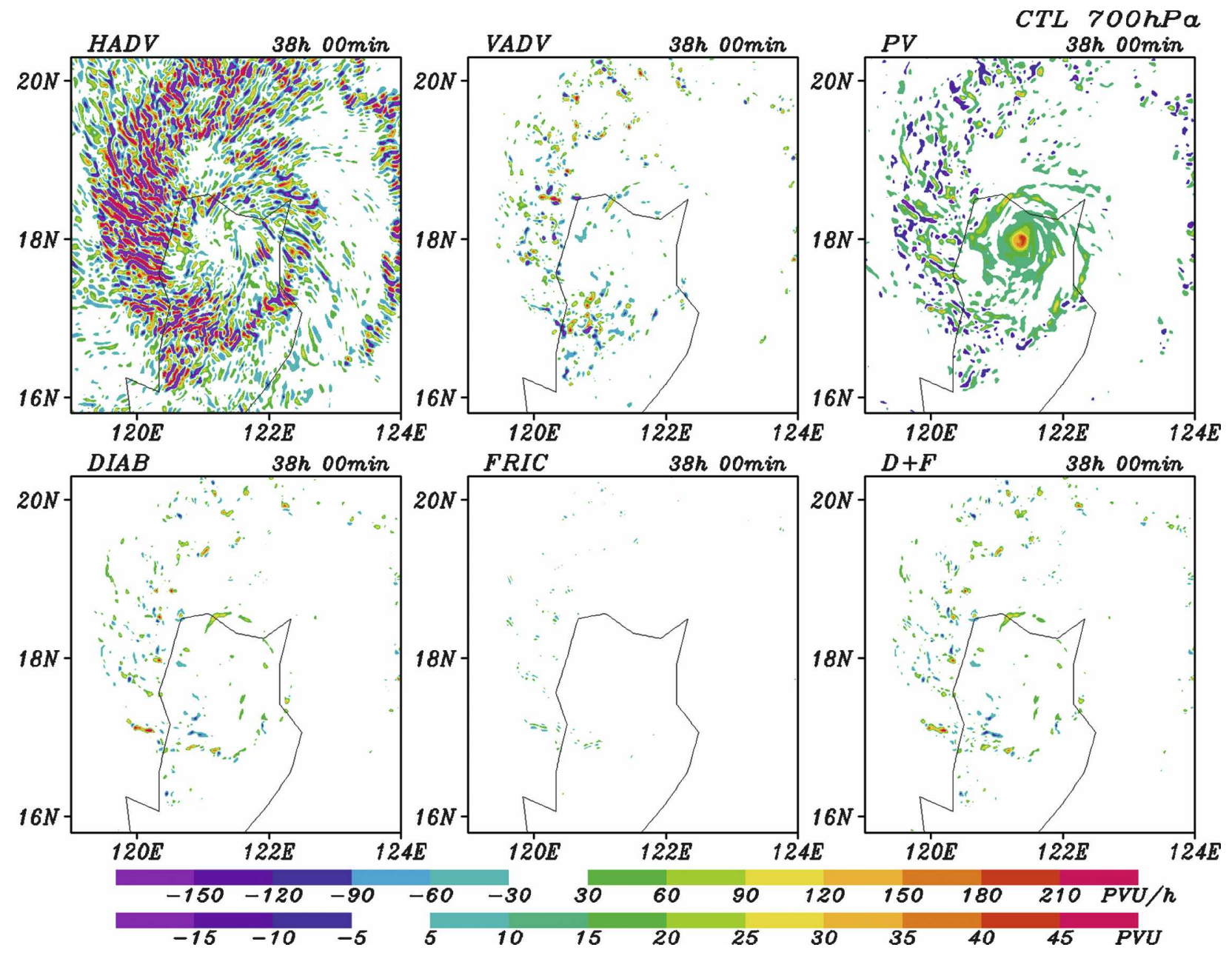

FIG. 14. The local changes of PV (as scaled by the upper color bar; PVU $\mathrm{h}^{-1}$ ) at $38 \mathrm{~h}, 700 \mathrm{hPa}$ in CTL due to the horizontal (HADV) and vertical (VADV) advection of PV, diabatic heating (DIAB, including the condensational and radiative heating), and friction (FRIC); $D+F$ denotes the sum of diabatic heating and friction and PV denotes the potential vorticity (as scaled by the lower color bar; PVU).

in the lower-middle troposphere (especially between $700-500 \mathrm{hPa}$; Fig. 14). Therefore, PV in the rainbands intensifies as a result of enhanced moist convection and is axisymmetrized by the lower-to-midlevel shear deformation flow of the storm and organized into a new convective eyewall with elevated PV (Wang 2008b). When the inner eyewall finally dissipates, the larger eyewall appears. As shown in the PV budget analyses at $925 \mathrm{hPa}$ at $54 \mathrm{~h}$ (Fig. 15), the diabatic heating is the leading factor contributing to the PV generation in the lower troposphere when the storm is located over the open ocean. Again, this analysis highlights the importance of the PV source from diabatic processes in the full-physics simulation. In CTL, the outer PV annulus forms slightly before the appearance of the larger eyewall in the wind field (Figs. 4a and 10). In NLT, the formation of the complete outer PV annulus appears after the formation of the larger eyewall in the wind field (Figs. $4 \mathrm{~b}$ and 12). Therefore, the mountains of Luzon hasten the weakening of the original eyewall and the development of the new large outer eyewall.

\section{Maintenance of the eyewall PV annulus}

The evolution of PV in the eyewall is highly influenced by the effects of surface friction and diabatic heating. Therefore, moist convection and friction must be important to the evolution of the PV annulus in the full-physics simulations discussed in section 3. The large PV annulus satisfies the necessary condition for barotropic instability. Such instability was studied by Schubert et al. (1999) and Kossin and Schubert (2001) using a barotropic model (in which vorticity evolution is stud- 


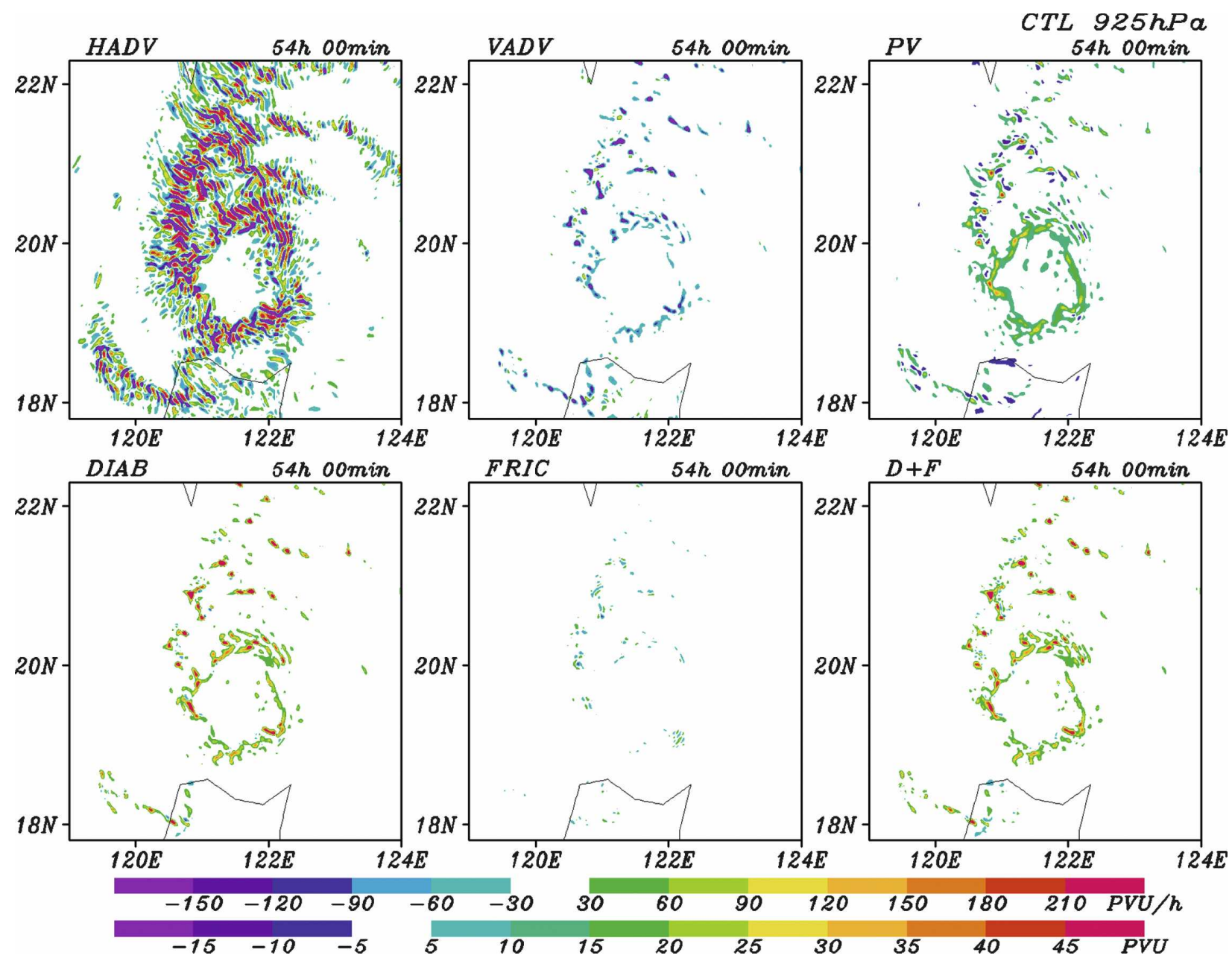

FIG. 15. As in Fig. 14, but for $925 \mathrm{hPa}$ at $54 \mathrm{~h}$.

ied instead of PV). They showed that depending on the ratio of the width of the vorticity annulus to the radius of the annulus at the initial state, the end state due to the release of the instability is either vorticity crystals [see Fig. 4 of Kossin and Schubert (2001), in which several patches of high-vorticity blobs are present] or a monopole. Therefore, without moist and frictional processes, a narrow large-PV annulus cannot maintain itself, and it breaks down into PV crystals (dynamically equivalent to vorticity crystals in the barotropic model as mentioned above) or evolves into monopole. To better understand the roles of the moist and frictional processes in the maintenance of the new eyewall PV annulus, several sensitivity experiments are conducted with or without moist processes, PBL processes, or surface heat flux (Table 1). All the sensitivity experiments start with a PV annulus with a large radius and a small $\mathrm{PV}$ patch in the center of the annulus, taken from the output of CTL at $54 \mathrm{~h}$ (Fig. 10g), and are integrated with the lateral boundary condition from the 54-72-h output of CTL. Each sensitivity experiment is integrated for $18 \mathrm{~h}$ on domain 4 (with 2-km horizontal grid spacing). As noted in section 2, in these sensitivity experiments some level of imbalance is introduced because of the inconsistencies in the model physics with both the initial and lateral boundary conditions. Nevertheless, these imbalances and inconsistencies are expected not to overtake the actual physics that we intend to explore within the relatively short integration time examined.

Without the moist and frictional processes in experiment DFL (dry and frictionless; Fig. 16), the high-PV annulus breaks down to form several small PV patches of different sizes and strengths within $1 \mathrm{~h}$ of integration (cf. Figs. 10g and 16a). The central high-PV patch migrates toward the inner edge of the annulus in the following hours and then orbits cyclonically with the other PV patches (Figs. 16b-d). The original central PV patch 
(a)

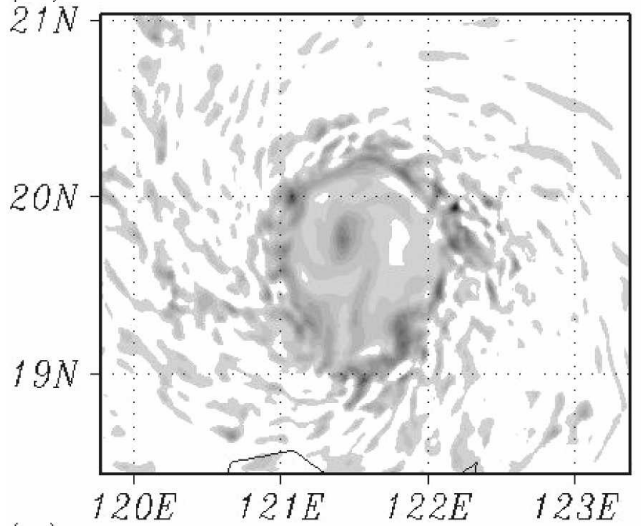

(c)

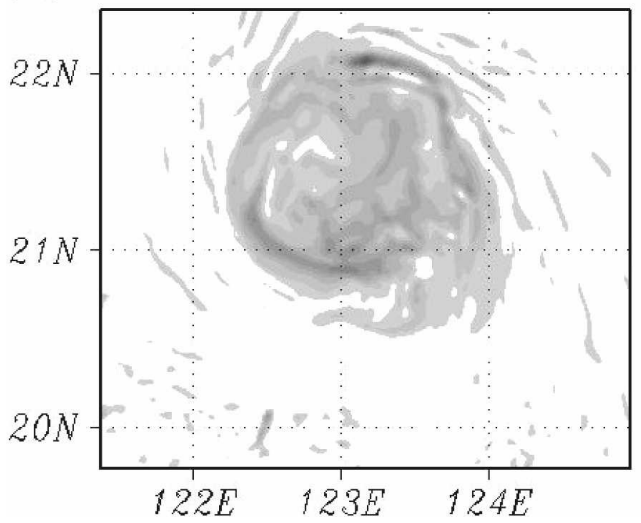

(b)
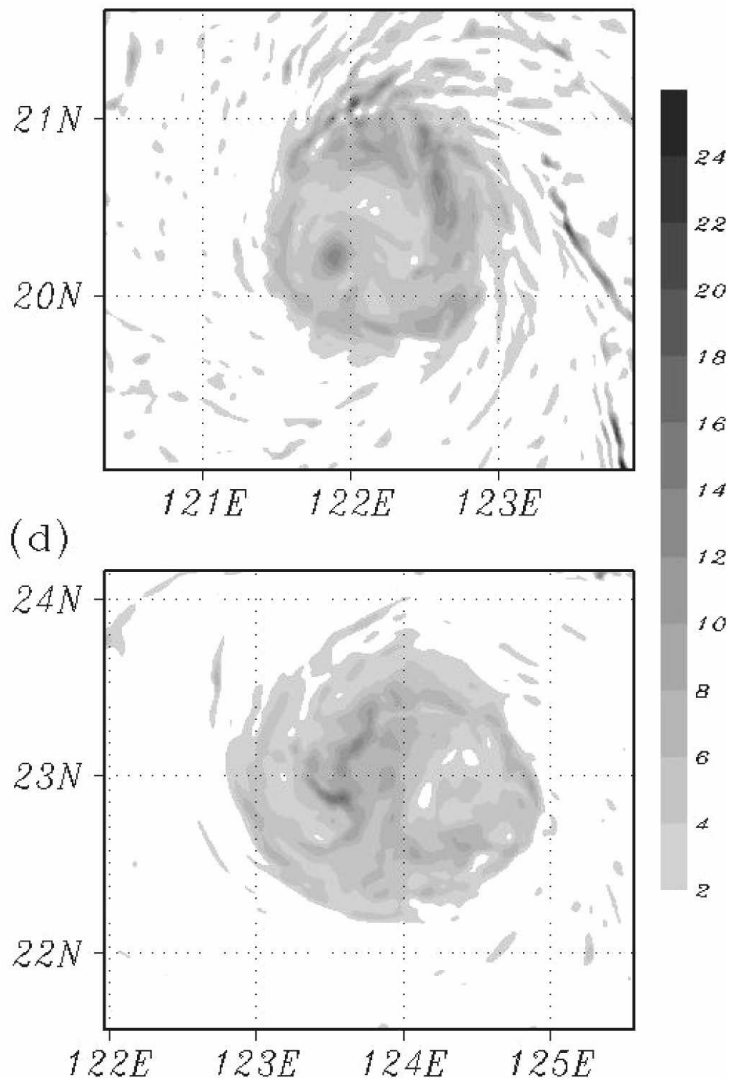

FIG. 16. PV (PVU) at $\sigma=0.875$ in DFL at (a) 1 , (b) 6, (c) 12, and (d) $18 \mathrm{~h}$.

decays while orbiting. At $t=9 \mathrm{~h}$, the PV patch and other mesovortices on the annulus have merged with one another to form larger mesovortices that are connected to PV filaments (figure not shown). Subsequently, only two major mesovortices remain at $t=18$ h. The central pressure fills from 962 to $971 \mathrm{hPa}$ throughout the 18-h integration. The distinctively different eyewall PV evolution in CTL and DFL demonstrates that the behavior of the eyewall evolution, which involves moist and frictional processes, could differ significantly from the unforced barotropic advective process.

Retaining PBL processes but excluding condensation heating in experiment DRY, the PV annulus exhibits a more rapid weakening (Fig. 17). The annulus is quickly distorted and rolls up into a number of small vortices in the first hour of integration (cf. Figs. 10g and 17a). As in DFL, the central high-PV patch migrates toward the proximity of the eyewall PV annulus and orbits cyclonically along the inner edge of the annulus. Merging and dissipation occur rapidly in the following hours. At $t=$ $6 \mathrm{~h}$, three major mesovortices and one weak mesovortex remain, which continue to dissipate and merge. Fi- nally, the mesovortices merge to form a monopole, which continues weakening throughout the end of the integration. The final central pressure is $991 \mathrm{hPa}, 29$ $\mathrm{hPa}$ higher than the central pressure of the initial vortex. Results from DFL and DRY thus indicate that the eyewall annulus cannot sustain itself without condensation heating and that surface friction is a sink to PV; it enhances the inward PV mixing by the boundary layer frictional inflow in the absence of condensation heating.

In experiment NHF (retaining both moist and frictional processes but with the surface heat fluxes turned off), the PV annulus is complete and undistorted in the first hour of integration (cf. Figs. 10g and 18a). The central high-PV patch orbits cyclonically along the inner edge of the annulus whereas the annulus starts to weaken and break down in the following hours (Fig. 18). The central PV patch collects high PV and strengthens while orbiting and the eyewall PV annulus weakens at the same time. It is not until the whole annulus disappears that the PV patch starts to weaken (Fig. 18c). By $t=18 \mathrm{~h}$, an approximate monopole forms; it has much larger central PV than in DRY but 
(a)

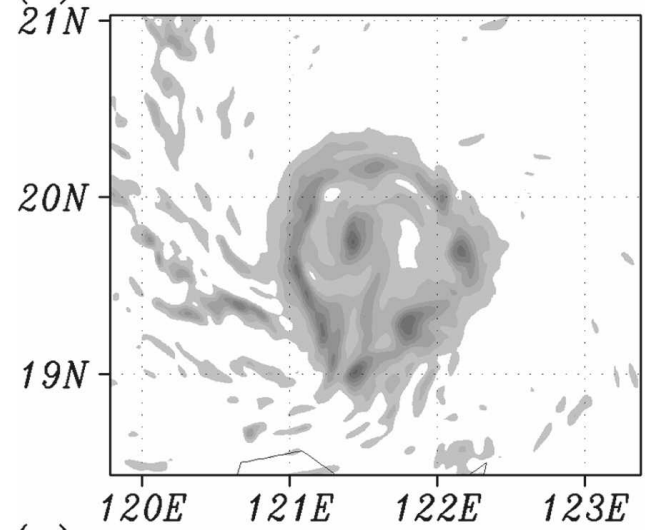

(c)

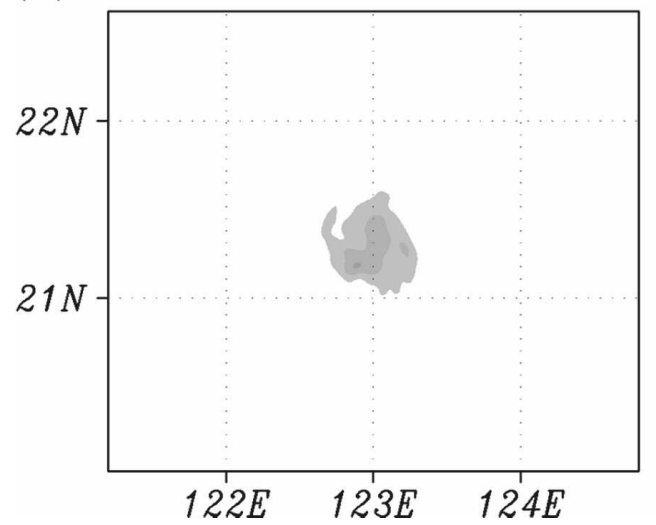

(b)

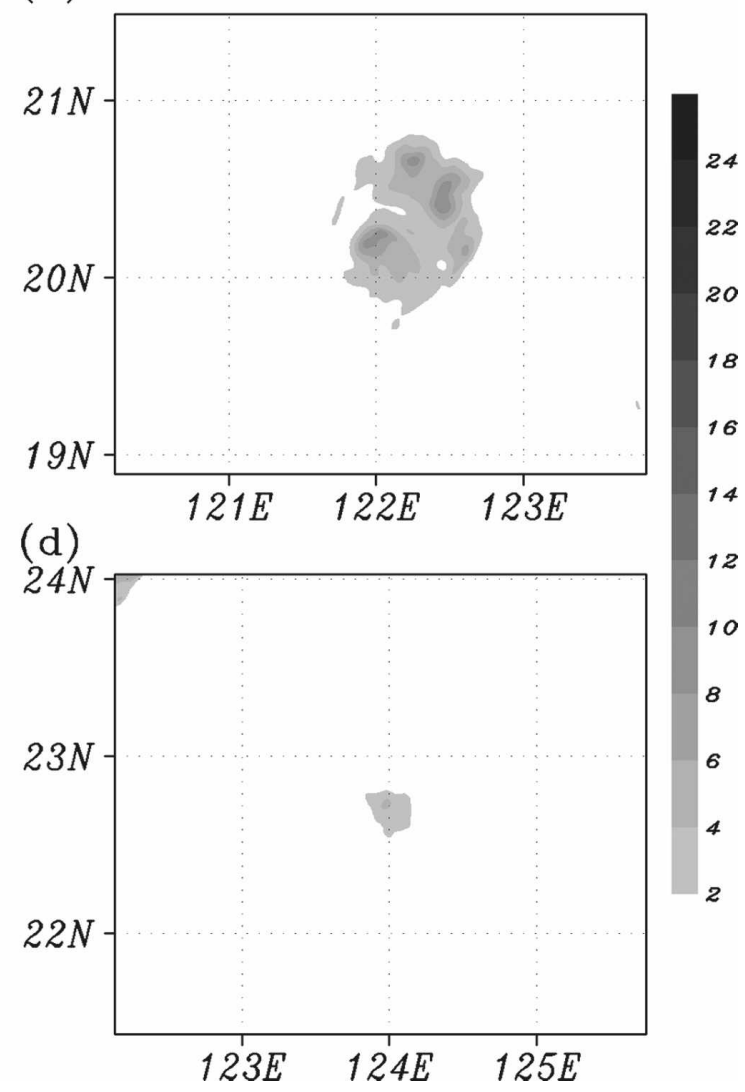

FIG. 17. As in Fig. 16, but for DRY.

much smaller PV than in the eyewall in CTL. In NHF, although moist processes are retained, diabatic heating is much smaller than in CTL because of the lack of moisture supply from the ocean underlying the eyewall. Diabatic heating in NHF is mainly associated with the moisture supply from the large-scale moisture convergence in the lower troposphere and acts as source of additional PV compared to DRY. The central pressure increases by $11 \mathrm{hPa}$ in the 18 -h integration.

If only the PBL process is turned off in the model (FL), a nearly complete PV annulus exists with a weak PV patch near the center at $t=1 \mathrm{~h}$ (cf. Figs. $10 \mathrm{~g}$ and 19a). In the following hours, the annulus breaks down into small mesovortices and the central PV patch is advected along the inner edge of the annulus. Then the small mesovortices merge to form larger mesovortices, and each larger mesovortex is connected to filaments of high PV (Fig. 19). At $t=15 \mathrm{~h}$, three major mesovortices coexist with some small PV patches and PV filaments (figure not shown). The three mesovortices do not merge throughout the remaining integration. The final central pressure is $952 \mathrm{hPa}, 10 \mathrm{hPa}$ lower than the initial central pressure because of the lack of any dissipation in this run. Comparing the PV evolution in CTL and FL, we can see that frictional processes play a role in narrowing the eyewall PV annulus and allowing the mesovortices to escape from merging with their neighbors in the presence of strong eyewall convection. This can be explained by the fact that friction is responsible for the low-level convergence in the eyewall. This frictional convergence serves as a stretching deformation and narrows the eyewall ascent and vorticity ring, thus maintaining the eyewall PV annulus.

The results presented in this section demonstrate that both surface friction and diabatic heating play quite important roles in the evolution of the typhoon eyewall. The convective heating is strongly coupled with the cyclonic PV anomalies. In our sensitivity experiments, the monopolar structure is most likely to appear in the absence of the moist process or the surface heat fluxes. These results also support the idea that the surface friction enhances the inward PV mixing by the frictionally induced boundary layer inflow. With full moist processes included, the eyewall PV appears to maintain an annular structure. In other words, the heating from moist convection would prevent the eyewall annulus 
(a)

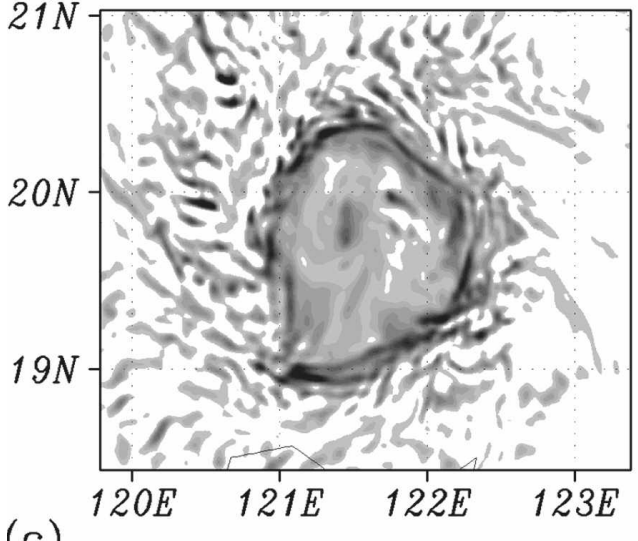

(c)

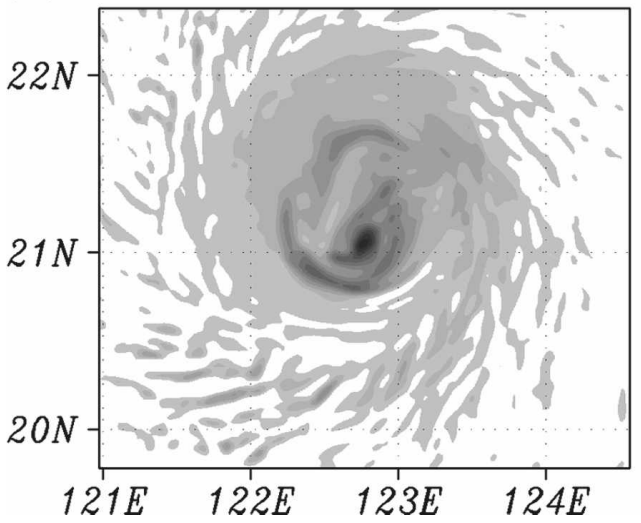

(b)
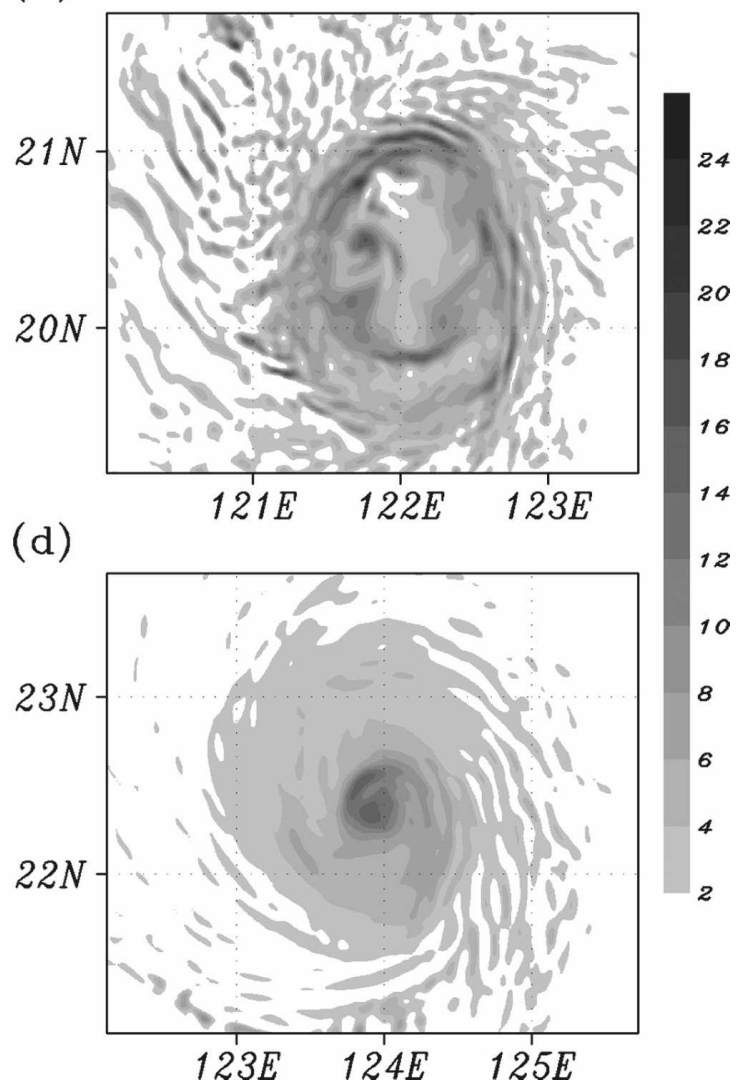

FIG. 18. As in Fig. 16, but for NHF.

from breaking down because of the barotropic instability regardless of the presence of friction.

It should be noted that the MM5 model employs the diffusion terms in both momentum (horizontal and vertical) and thermodynamic equations. One may ask whether the diffusion in the current model is much larger than that in the barotropic model, thus providing an unfair comparison. It is believed that the diffusion should not be the major reason for the differences between the barotropic experiment and the full-physics experiment. Even if the same diffusion were used in the barotropic model as in MM5, the PV ring still could not be maintained because of the intrinsic instability and lack of a PV source to enhance the PV ring. As in the experiments without condensation heating (i.e., DFL and DRY), the PV ring is no longer maintained and disintegrates.

However, if the sensible and latent heat fluxes from the underlying surface (i.e., the fuel for moist convection) are turned off, barotropic instability of the eyewall may ruin the PV annulus quickly and result in the formation of a monopolar PV structure. Therefore, moist convection in the eyewall is critical for the maintenance of the eyewall PV annulus. Our results support the notion of Schecter and Montgomery (2007), who show that moist processes may play a role in stabilizing the TC vortex by suppressing the growth rate of unforced unstable modes.

For a landfalling TC, the sensible and latent heat fluxes from the underlying surface decrease tremendously, resulting in the weakening of the eyewall convection and diminishing the PV anomaly production from condensation. Moreover, the increased surface friction acts to enhance the inward PV mixing in the absence of strong eyewall convection. As a result, the eyewall PV annulus of a landfalling typhoon tends to be destroyed, and then the enhanced mixing between the eyewall and the eye contributes to the formation of the monopole. However, in the presence of strong eyewall convection, as seen in CTL, frictional processes can help narrow the PV annulus and contribute to the maintenance of the PV annulus of the large eyewall. Therefore, frictional processes play a complicated (dual) role in affecting the evolution of eyewall PV. 
(a)

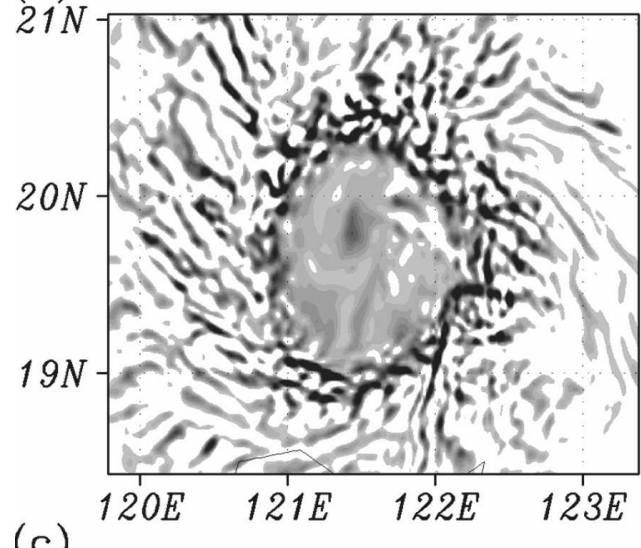

(c)

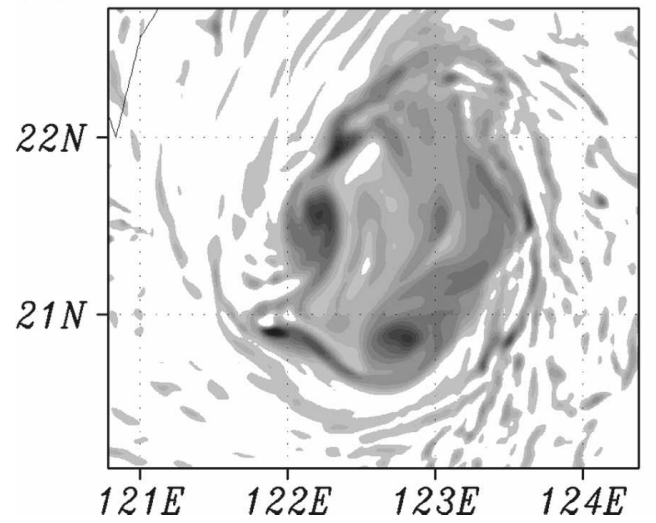

(b)
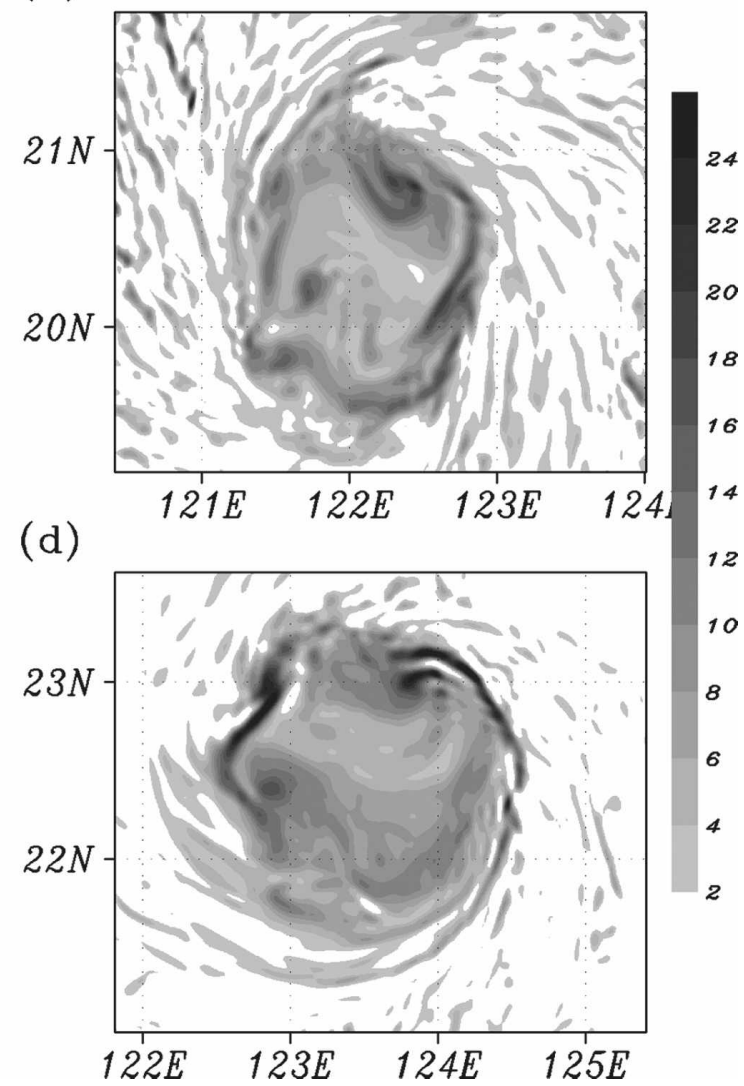

FIG. 19. As in Fig. 16, but for FL.

\section{Conclusions}

It is found that the breakdown and reformation of the eyewall of Zeb are largely controlled by the underlying surface. In the eyewall replacement process of Zeb, both the breakdown and reformation of the eyewall are closely related to the availability of the surface heat and moisture fluxes from the lower boundary.

The large eye occurs as inner eyewall dissipates and the outer eyewall intensifies. However, the eyewall replacement of Zeb is quite different from the conventional concentric eyewall replacement of Willoughby et al. (1982). In our case, the landfall of the eyewall triggers the particular eyewall replacement process. The weakening of the original eyewall convection is initiated by the reduction of surface moisture and heat fluxes after landfall rather than by the cutoff of moisture fluxes due to the development of the outer eyewall. The outer eyewall, developed over the ocean from outer spiral rainbands, intensifies because the moisture and heat fluxes from the underlying ocean are available for the outer circulation. Therefore, the presence of Luzon plays the key role in the eyewall evolution in this case. The mountain plays an extra role in the weakening and dissipation of the original eyewall and the early formation of a large outer eyewall by triggering strong outer spiral rainbands offshore.

It is believed that similar eyewall evolution may often occur when a typhoon encounters terrain of a size comparable to the size of the storm circulation. For example, when Hurricane Wilma (2005) encountered the Yucatán Peninsula, a large eyewall occurred when Wilma reentered the Caribbean Sea. The radar images show that the large eye appeared while the inner eyewall dissipated because of the landfall process. The intensity and size of the storm and the height and size of the terrain are all important factors that affect the eyewall evolution of landfalling typhoons.

It is also found that surface friction both acts to enhance the inward PV mixing by the frictionally induced boundary layer inflow in the absence of diabatic heating and also narrows the PV annulus through stretching deformation in the presence of strong eyewall convection. Diabatic heating is the PV source and acts to enhance the PV annulus. It is suggested that if the rate of $\mathrm{PV}$ production in the eyewall is more efficient than the 
rate at which the PV is advected or mixed away from the eyewall, the high-PV annulus can be maintained. In contrast to the results presented in Schubert et al. (1999) and Kossin and Schubert (2001), the very narrow high-PV annulus in our control full-physics experiment maintains its annular structure to the end of simulation. Results of sensitivity experiments indicate the importance of moist processes and the underlying surface conditions in the evolution of the typhoon eyewall.

There are many factors affecting the evolution of the eyewall, including the internal dynamics and environmental conditions (such as sea surface temperature, friction, environmental vertical shear, environmental heat or momentum sources, etc.). The numerical results in this study indicate that the availability of sensible and latent heat from the underlying surface is crucial for the eyewall and intensity evolution of a landfalling typhoon. Our results suggest that the presence of diabatic heating and friction lead to qualitatively quite different behaviors as compared to those based on purely advective dynamics. However, the detailed mechanism involved in the processes requires further investigation through more specifically designed numerical experiments. For example, how do friction, terrain, latent heat, and sensible heat interact with each other in the storm core and what is their relative importance in the eyewall evolution? What determines the efficiency of the symmetrization process? It is expected that a suite of idealized model simulations with full physics could be used to gain more physical insights into these interesting issues.

Acknowledgments. The leading author is supported by NSC94-2119-M-002-006-AP1, NSC95-2119-M-002039-MY2, and NTU-97R0302. YW has been supported in part by NSF Grants ATM-0427128 and ATM0754039 and ONR Grant N00014-06-10303. The authors wish to thank Jim Kossin, Chris Davis, and the anonymous reviewers for their valuable comments.

\section{REFERENCES}

Betts, A. K., and M. J. Miller, 1986: A new convective adjustment scheme. Part II: Single column tests using GATE wave, BOMEX, ATEX, and Arctic air-mass data sets. Quart. J. Roy. Meteor. Soc., 112, 693-709.

Black, M. L., and H. E. Willoughby, 1992: The concentric eyewall cycle of Hurricane Gilbert. Mon. Wea. Rev., 120, 947-957.

Blackadar, A. K., 1976: Modeling the nocturnal boundary layer. Preprints, Third Symp. on Atmospheric Turbulence and Air Quality, Raleigh, NC, Amer. Meteor. Soc., 46-49.

_ 1979: High-resolution models of the planetary boundary layer. Advances in Environmental Science and Engineering, J. Pfafflin and E. Ziegler, Eds., Vol. 1, Gordon and Breach, 50-85.
Brand, S., and J. W. Blelloch, 1973: Changes in the characteristics of typhoons crossing the Philippines. J. Appl. Meteor., 12, 104-109.

— and - 1974: Changes in the characteristics of typhoons crossing the island of Taiwan. Mon. Wea. Rev., 102, 708-713.

Chen, Y., and M. K. Yau, 2003: Asymmetric structures in a simulated landfalling hurricane. J. Atmos. Sci., 60, 2294-2312.

Emanuel, K. A., 1997: Some aspects of hurricane inner-core dynamics and energetics. J. Atmos. Sci., 54, 1014-1026.

Grell, G. A., J. Dudhia, and D. R. Stauffer, 1995: A description of the fifth-generation Penn State/NCAR Mesoscale Model (MM5). NCAR Tech. Note NCAR/TN-398+STR, 122 pp.

Kossin, J. P., and M. D. Eastin, 2001: Two distinct regimes in the kinematic and thermodynamic structure of the hurricane eye and eyewall. J. Atmos. Sci., 58, 1079-1090.

— patterns, and rapid pressure falls in hurricane-like vortices. $J$. Atmos. Sci., 58, 2196-2209.

Montgomery, M. T., and R. J. Kallenbach, 1997: A theory for vortex Rossby waves and its application to spiral bands and intensity changes in hurricanes. Quart. J. Roy. Meteor. Soc., 123, 435-465.

— tively forced vortex Rossby waves in a three-dimensional quasigeostrophic model. J. Atmos. Sci., 55, 3176-3207.

Reasor, P. D., M. T. Montgomery, F. D. Marks Jr., and J. F. Gamache, 2000: Low-wavenumber structure and evolution of the hurricane inner core observed by airborne dual-Doppler radar. Mon. Wea. Rev., 128, 1653-1680.

Reisner, J., R. J. Rasmussen, and R. T. Bruintjes, 1998: Explicit forecasting of supercooled liquid water in winter storms using the MM5 mesoscale model. Quart. J. Roy. Meteor. Soc., 124B, 1071-1107.

Schecter, D. A., and M. T. Montgomery, 2007: Waves in a cloudy vortex. J. Atmos. Sci., 64, 314-337.

Schubert, W. H., and J. J. Hack, 1982: Inertial stability and tropical cyclone development. J. Atmos. Sci., 39, 1687-1697.

—, M. T. Montgomery, R. K. Taft, T. A. Guinn, S. R. Fulton, J. P. Kossin, and J. P. Edwards, 1999: Polygonal eyewalls, asymmetric eye contraction, and potential vorticity mixing in hurricanes. J. Atmos. Sci., 56, 1197-1223.

Shapiro, L. J., and H. E. Willoughby, 1982: The response of balanced hurricanes to local sources of heat and momentum. $J$. Atmos. Sci., 39, 378-394.

Wang, Y., 2002a: Vortex Rossby waves in a numerically simulated tropical cyclone. Part I: Overall structure, potential vorticity, and kinetic energy budgets. J. Atmos. Sci., 59, 1213-1238.

— 2002b: Vortex Rossby waves in a numerically simulated tropical cyclone. Part II: The role in tropical cyclone structure and intensity changes. J. Atmos. Sci., 59, 1239-1262.

_ 2008a: Structure and formation of an annular hurricane simulated in a fully compressible, nonhydrostatic modelTCM4. J. Atmos. Sci., 65, 1505-1527.

_ 2008b: Rapid filamentation zone in a numerically simulated tropical cyclone. J. Atmos. Sci., 65, 1158-1181.

- , and C.-C. $\mathrm{Wu}, 2004$ : Current understanding of tropical cyclone structure and intensity changes-A review. Meteor. Atmos. Phys., 87, 257-278.

Willoughby, H. E., 1990: Temporal changes of the primary circulation in tropical cyclones. J. Atmos. Sci., 47, 242-264.

, and P. G. Black, 1996: Hurricane Andrew in Florida: Dynamics of a disaster. Bull. Amer. Meteor. Soc., 77, 543-549.

_ J. A. Clos, and M. G. Shoreibah, 1982: Concentric eye walls, 
secondary wind maxima, and the evolution of the hurricane vortex. J. Atmos. Sci., 39, 395-411.

Wu, C.-C., 2001: Numerical simulation of Typhoon Gladys (1994) and its interaction with Taiwan terrain using the GFDL hurricane model. Mon. Wea. Rev., 129, 1533-1549.

— mechanisms of hurricane-environment interaction on hurricane movement from the potential vorticity perspective. $J$. Atmos. Sci., 53, 2264-2282.

— , and Y. H. Kuo, 1999: Typhoons affecting Taiwan-Current understanding and future challenges. Bull. Amer. Meteor. Soc., 80, 67-80.

—, T.-H. Yen, Y.-H. Kuo, and W. Wang, 2002: Rainfall simulation associated with Typhoon Herb (1996) near Taiwan. Part I: The topographic effect. Wea. Forecasting, 17, 10011015.

_ K.-H. Chou, H.-J. Cheng, and Y. Wang, 2003a: Eyewall contraction, breakdown, and reformation in a landfalling typhoon. Geophys. Res. Lett., 30, 1887, doi:10.1029/2003GL017653.

, T.-S. Huang, W.-P. Huang, and K.-H. Chou, 2003b: A new look at the binary interaction: Potential vorticity diagnosis of the unusual southward movement of Typhoon Bopha (2000) and its interaction with Supertyphoon Saomai (2000). Mon. Wea. Rev., 131, 1289-1300.

and K.-H. Chou, 2004: Potential vorticity diagnosis of the key factors affecting the motion of Typhoon Sinlaku (2002). Mon. Wea. Rev., 132, 2084-2093.

Wu, L., and S. A. Braun, 2004: Effects of environmentally induced asymmetries on hurricane intensity: A numerical study. $J$. Atmos. Sci., 61, 3065-3081.

Yang, B., Y. Wang, and B. Wang, 2007: The effect of internally generated inner-core asymmetries on tropical cyclone potential intensity. J. Atmos. Sci., 64, 1165-1188.

Yeh, T.-C., and R. L. Elsberry, 1993a: Interaction of typhoons with the Taiwan orography. Part I: Upstream track deflections. Mon. Wea. Rev., 121, 3193-3212.

$\longrightarrow$, and 1 1993b: Interaction of typhoons with the Taiwan orography. Part II: Continuous and discontinuous tracks across the island. Mon. Wea. Rev., 121, 3213-3233.

Zhang, D., and R. A. Anthes, 1982: A high-resolution model of the planetary boundary layer-Sensitivity tests and comparisons with SESAME-79 data. J. Appl. Meteor., 21, 1594-1609. 